University of South Florida

DIGITAL COMMONS

Digital Commons @ University of

@ UNIVERSITY OF SOUTH FLORIDA

South Florida

School of Geosciences Faculty and Staff

Publications

School of Geosciences

$5-1991$

\title{
An Introduction to the Global Positioning System and Some Geological Applications
}

Timothy H. Dixon

Jet Propulsion Laboratory, Pasadena, CA, thd@usf.edu

Follow this and additional works at: https://digitalcommons.usf.edu/geo_facpub

Part of the Earth Sciences Commons

\section{Scholar Commons Citation}

Dixon, Timothy H., "An Introduction to the Global Positioning System and Some Geological Applications" (1991). School of Geosciences Faculty and Staff Publications. 504.

https://digitalcommons.usf.edu/geo_facpub/504

This Article is brought to you for free and open access by the School of Geosciences at Digital Commons @ University of South Florida. It has been accepted for inclusion in School of Geosciences Faculty and Staff Publications by an authorized administrator of Digital Commons @ University of South Florida. For more information, please contact digitalcommons@usf.edu. 


\title{
AN INTRODUCTION TO THE GLOBAL POSITIONING SYSTEM AND SOME GEOLOGICAL APPLICATIONS
}

\author{
T. H. Dixon \\ Jet Propulsion Laboratory \\ Division of Earth and Planetary Sciences \\ Pasadena, California
}

\begin{abstract}
Receivers equipped to measure dual frequency carrier phase signals from satellites of the Global Positioning System (GPS) have been capable, under special conditions, of determining relative horizontal positions among stations separated by one to a few hundred kilometers with a precision of one to several millimeters since the early 1980s. The major obstacles to making this capability routine, extending it to all parts of the globe, and extending it to longer station separations, have been equipment cost, limitations in the GPS satellite constellation, arduous data analysis, uncertainties in satellite orbits, uncertainties in propagation delays associated with variable tropospheric water vapor, and difficulties in resolving carrier phase cycle ambiguities. Recent improvements have occurred in all these areas. The increasing ease and reduced cost of GPS
\end{abstract}

data acquisition and analysis are having a significant impact on studies of near-fault crustal deformation and earthquake processes, until recently the province of conventional terrestrial geodetic techniques. The enhanced satellite constellation, improved models, and establishment of global tracking networks have extended several millimeters horizontal positioning capability to station separations of $1000 \mathrm{~km}$ or more in virtually all parts of the world. This enables study of new classes of tectonic problems that previously were difficult to attack with any geodetic technique. Examples include a complete kinematic description of ongoing crustal deformation in broad, complex continental plate boundary zones, and measurement of relative plate motion at convergent boundaries where global models may be poorly constrained.

\section{INTRODUCTION}

With the advent in the early 1980 s of a satellite-based navigation system known as the Global Positioning System (GPS) operated by the U.S. Department of Defense it became possible for a user with the proper receiver to obtain almost instantaneous three-dimensional position information accurate to several meters. With the completion of the satellite constellation in the early 1990s this capability will be extended to virtually all parts of the globe. This is a remarkable achievement and builds on a number of technological advances in the last several decades. Even more remarkable is the fact that with careful attention to experiment configuration and data analysis it is possible to obtain relative position data 3 orders of magnitude more precise than the design level of the system. This enhanced performance allows for measurement of crustal strain and fault motion rates in just a few years.

This paper reviews fundamental principles of GPS, discusses some geological and geophysical applications and their accuracy requirements, and considers implications for GPS experiment design. Recent developments extending the range and accuracy of GPS measurements are emphasized. I have aimed for broad coverage of most of the relevant topics and an intuitive rather than complete or rigorous treatment, giving more detailed references where appropriate.

\section{A BRIEF HISTORY OF THE GPS PROGRAM}

The space segment of GPS is a constellation of satellites in high Earth orbit equipped with powerful radio frequency transmitters and highly stable atomic clocks. Easton [1978] reviews the major developments leading to this capability. In 1967 an early prototype of a GPS satellite known as Timation 1 was launched into low Earth orbit $(\sim 900 \mathrm{~km}$ altitude) as part of a military test program in satellite navigation. Weighing about $40 \mathrm{~kg}$ and consuming only 6 W of power, it carried a UHF transmitter slaved to a stable quartz clock, with a frequency drift of several parts in $10^{11}$ per day. Additional proof-of-concept satellites followed, culminating 10 years later in the Navigation Technology Satellite (NTS) 2, very similar to subsequent GPS satellites. NTS-2 was launched into a $20,300-\mathrm{km}$-altitude 
orbit, weighed $440 \mathrm{~kg}$, consumed $400 \mathrm{~W}$ of power, and transmitted two $L$ band $(\sim 1.2$ and $1.5 \mathrm{GHz})$ timing and ranging signals based on a sophisticated cesium clock, with a frequency drift of less than two parts in $10^{13}$ per day. One year after NTS-2, the first "Block 1" GPS satellite was launched, part of the operational test phase of the GPS program. By 1990 the Block 1 constellation included six functioning satellites launched between 1978 and 1985.

It has been recognized for some time that high-precision geodetic measurements could be made by exploiting signals from artificial satellites [e.g., Preston et al., 1972; MacDoran, 1979; Counselman and Shapiro, 1979]. The Block 1 constellation has proven satisfactory for developing and refining experiment design and analytical concepts and for initiating a number of high-precision geodetic monitoring programs. The first Block 2 satellite, with a number of improvements relative to its forebears, was launched in February 1989. As of this writing, a total of 10 Block 2 satellites are in operation. A total of 21 Block 2 satellites plus three spares are planned to be in operation by the end of 1992 . They will orbit at an altitude of about $20,000 \mathrm{~km}$ in six orbital planes with 12-hour periods, enabling simultaneous observation of four or more GPS satellites in virtually all parts of the globe.

\section{POINT POSITIONING WITH GPS}

\section{Range Measurement}

An observer on Earth can uniquely locate his position by determining the distance between himself and three satellites whose orbital positions are already known. With GPS, distance information is based on the travel time $\tau$ of a satellite signal, obtained by measuring the difference between the transmit $\left(t_{s}\right)$ and receive $\left(t_{r}\right)$ times at the GPS receiver of a special ranging code, described in the next section. If we ignore transmission media effects on the speed of light $c$ and any timing (clock) errors, then the true range $\rho$ between satellite and receiver is just $c\left(t_{r}-t_{s}\right)$. Errors in receiver or satellite clocks are present in the range estimate, which for this reason is referred to as pseudorange $R$, defined more precisely as

$$
R=p+c\left(\Delta t_{r}-\Delta t_{s}+\Delta t_{p}\right)
$$

where $\Delta t_{r}$ is the receiver clock offset from "true" (GPS system) time (we ignore any other receiver-induced errors), $\Delta t_{s}$ is the satellite clock offset, and $\Delta t_{p}$ is the delay associated with all other error sources, mainly due to atmospheric propagation effects. Information from a fourth satellite allows a first-order clock correction $\left(\Delta t_{r}-\Delta t_{s}\right)$, and approaches discussed below can be applied to estimate and correct for $\Delta t_{p}$, enabling meter-level positioning under ideal conditions.

Observation geometry affects the quality of the resulting three-dimensional position. Satellites that appear close to one another in the sky provide correlated (redundant) range information, an effect known as geometric dilution of precision (GDOP). If observations are limited to four satellites by receiver design, these geometric effects can be minimized by choosing satellites which maximize the volume of a tetrahedron, defined by the points of intersection on a unit sphere centered on the user, of vectors between the satellite and the ground receiver.

It is also possible to obtain distance information (strictly speaking, the change in distance) through phase measurements on the carrier signal itself, keeping track of the number of cycles after signal acquisition. Assuming perfect clocks, and ignoring propagation effects,

$$
\begin{aligned}
\rho & =n \lambda+\phi \lambda \\
& =\left(v_{\phi} / f\right)(n+\phi)
\end{aligned}
$$

where $n$ is the number of integer carrier wavelengths at signal acquisition (initially unknown), $\phi$ is the phase in cycles, $\lambda$ is the wavelength, $f$ is the frequency, and $v_{\phi}$ is the phase velocity (the importance of distinguishing $v_{\phi}$ and group velocity, $v_{g}$, will become apparent). Since the wavelength of the carrier is considerably shorter than that of the lower frequency code modulations (Table 1), the resulting "distance" measurement, though ambiguous by the initial number of wavelengths, is considerably more precise than a pseudorange measurement and is one of the keys to high-precision GPS measurements [Bossler et al., 1980; Counselman and Gourevitch, 1981; Remondi, 1985]. Carrier phase is not measured directly, as this would require very high sampling rates. Rather, the signal is mixed ("heterodyned") with a signal generated by the receiver's internal clock (local oscillator) and, after band-pass or low-pass filtering, the resulting lower frequency "carrier beat phase" is sampled. Most current generation receivers accomplish this "downconversion" with electronics that include extensive analog circuitry. Some newer generation receivers have largely digital architecture, reducing production cost, size, and power consumption and enabling digital sampling of the carrier phase signal with only minimal preprocessing [Melbourne,

\begin{tabular}{|c|c|c|c|c|}
\hline & \multicolumn{2}{|c|}{ Carriers } & \multicolumn{2}{|c|}{ Code Modulations } \\
\hline & $L I$ & $L 2$ & $P$ & $\begin{array}{c}C / A \\
\text { (L1 only) }\end{array}$ \\
\hline Frequency (carrier) & 1.57542 & 1.2276 & 10.23 & 1.023 \\
\hline $\begin{array}{l}\text { or chip rate } \\
\text { (code modulation) }\end{array}$ & $\mathbf{G H z}$ & $\mathrm{GHz}$ & $\mathrm{MHz}$ & $\mathrm{MHz}$ \\
\hline Wavelength & $19.0 \mathrm{~cm}$ & $24.4 \mathrm{~cm}$ & $-30 \mathrm{~m}$ & $\sim 300 \mathrm{~m}$ \\
\hline
\end{tabular}
1990].

TABLE 1. Summary of GPS Signal Characteristics

For analysis purposes we consider the pseudorange or phase parameters in terms of what the receiver actually sees (the "observable"), explicitly accounting for major 
error sources. For example, in simplified form the phase observable, sometimes called integrated Doppler or accumulated delta range, can be written (in units of cycles) for a single receiver-satellite pair as

$$
\phi(t)=-f \tau(t)+v(t)+a
$$

where the delay $\tau(=\rho / c)$ is the geometric delay due only to the satellite-station geometry, ignoring propagation effects, $v$ represents errors ("noise") including propagation effects, and $a$ is a constant, representing a combination of the carrier phase cycle ambiguity and an initial phase offset between satellite and receiver oscillators. Equation (3) ignores time-dependent clock errors in both the satellite and receiver; also, the various effects represented by $v$ and $a$ must be considered in more detail. King et al. [1985] and Leick [1990] give a complete derivation of the phase observable equations.

\section{Signal Structure}

The following discussion is abbreviated from Spilker [1978]. GPS satellites transmit two $L$-band carrier frequencies, each modulated by several lower frequency signals (Table 1). The carriers ( $L 1$ at $1.57542 \mathrm{GHz}$ and $L 2$ at $1.22760 \mathrm{GHz}$ ) are coherent multiples of a $10.23-\mathrm{MHz}$ atomic clock, a stable oscillator that provides a frequency standard on each satellite $(L 1=154 \times 10.23 \mathrm{MHz}, L 2=$ $120 \times 10.23 \mathrm{MHz}$ ). The clock frequency is actually set slightly lower than this nominal frequency to account for relativistic effects [McCaskill and Buisson, 1985] so that an observer on the ground "sees" $10.23 \mathrm{MHz}$ almost exactly.

The $L 1$ carrier has two components. The "inphase" component is modulated by the precision $(P)$ code. A lower frequency coarse/acquisition (C/A) code is modulated in quadrature, i.e., on the same carrier frequency shifted $90^{\circ}$ (Figure 1). The $L 2$ carrier is normally modulated only with the $P$ code. All three carriers also are modulated with a low bit rate $(50 \mathrm{~Hz})$ data stream transmitting satellite health, ephemeris, and other housekeeping information. These codes, $P(t), C(t)$, and $D(t)$, can be considered as square waves with values of \pm 1 and are termed pseudorandom noise (PRN) codes because they have sufficiently long repeat times that they appear random to a user without knowledge of code structure. For example, the $P$ code repeat time is 37 weeks. Each satellite is assigned a unique 1-week portion of the code, and, since the number of active satellites will not exceed 24, each satellite can be uniquely identified by a PRN number corresponding to the code portion transmitted.

We can represent $L 1$ and $L 2$ signals as

$$
\begin{aligned}
S 1(t) & =A_{P 1} P(t) D(t) \cos \left(\omega_{1} t\right) \\
& +A_{c} C(t) D(t) \sin \left(\omega_{1} t\right) \\
S 2(t) & =A_{P 2} P(t) D(t) \cos \left(\omega_{2} t\right)
\end{aligned}
$$

where $\omega_{1}$ and $\omega_{2}$ are the angular frequencies $(\omega=2 \pi f)$ associated with the $L 1$ and $L 2$ carriers and $A_{p}$ and $A_{c}$ are the relative amplitudes of the $P$ and $C / A$ codes. The amplitude of the $C / A$ signal is higher to facilitate initial signal acquisition. One effect of the PRN code modulations on the carriers is to spread the energy of the $P$ code signal $\pm 10.23 \mathrm{MHz}$ around the carrier center frequency; because of this wide bandwidth the GPS signal is often referred to as "spread spectrum." Figure 1 illustrates schematically how a carrier signal is biphase modulated with a PRN code. A receiver with knowledge of the code structure and an internal clock can recover an estimate of signal transit time by cogenerating the code sequence and performing a cross correlation between the received signal and its internal code, determining the time delay necessary to match the two sequences.

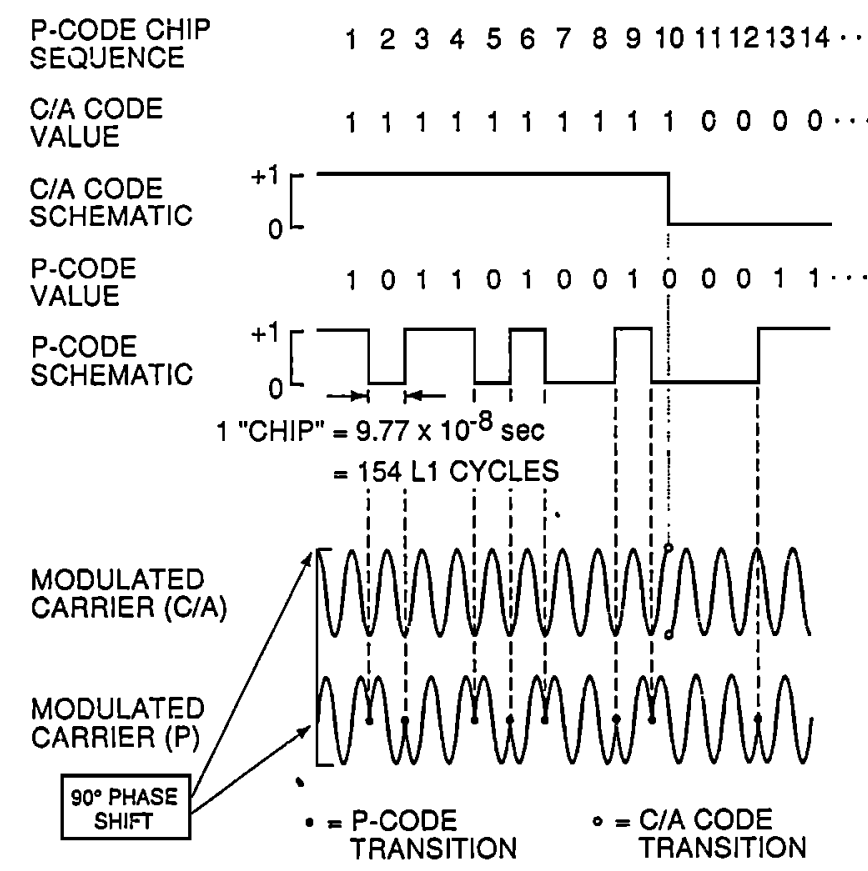

Figure 1. Schematic illustration of biphase and quadrature modulation of the $L 1$ carrier signal $(1575.42 \mathrm{MHz}$ ) by the $P$ $(10.23 \mathrm{MHz})$ and $C / A(1.023 \mathrm{MHz})$ pseudorandom noise (PRN) codes. Small circles on modulated carriers denote phase inversion points. Note $90^{\circ}$ phase shift between $P$ and $C / A$ modulations. In this illustration there is only one carrier cycle per $P$ code chip. In actuality there are $154 L 1$ cycles per $P$ code chip.

Receiver design affects the type of observable that can be extracted from the spectrum of GPS signals. There are currently two basic receiver architectures in common use for high-precision geodesy, code correlating (as described above) and codeless, where only the carrier phase observable is available. Code-correlating receivers can recover ("reconstruct") the phase observable as a by-product of the correlation process. Recovery of carrier phase without code knowledge requires a nonlinear detection scheme such as signal squaring. In effect, the signal is multiplied by itself, making the original phase inversions (equivalent to an 
amplitude change of \pm 1 ) unity, giving the second harmonic of the carrier with no code modulations at double the original frequency, or half the wavelength. The advantage of the codeless approach is that the high-precision part of the signal (the carrier phase) can be utilized without knowledge of the classified $P$ code, which may not be available in the future. Disadvantages include reduction of signal-to-noise ratio, possibly important under marginal observing conditions such as periods of high ionospheric activity, and reduced effective wavelength, making carrier phase cycle ambiguity resolution more difficult. Also, $P$ code data, which are otherwise useful for clock synchronization, data editing, and, depending on data quality, resolution of carrier phase cycle ambiguities, are obviously unavailable. Some civilian receivers employ a hybrid approach, with code-correlating capability on $L 1$ to recover the nonclassified $C / A$ code for clock synchronization and navigation information, and a codeless channel to recover the second harmonic of $L 2$.

Block 2 satellites have additional security features that will affect civilian users. "Selective availability" (SA) reduces point positioning accuracy to about $100 \mathrm{~m}$ by reducing the accuracy of the broadcast ephemeris, altering the clock epoch, and dithering the clock frequency, thus affecting both code-correlating and codeless receivers. "Antispoofing" (AS) will be activated periodically for test purposes and consists of encryption of the $P$ code; this would not affect codeless receivers. Most aspects of SA and AS will not cause serious impact on high-precision geodetic applications. However, if activated, AS would limit high-precision dynamic applications, and one important aspect of SA (clock dithering) is discussed below.

\section{HIGH-PRECISION GEODESY: RELATIVE POSITIONING}

Uncertainties in a GPS point position may be several meters to several tens of meters, although Malys and Jensen [1990] recently reported point position uncertainties of about $1 \mathrm{~m}$ using data from a specially configured global experiment. One source of uncertainty in a GPS point position is the inherent imprecision of the $P$ code group delay measurement, meter level for most receivers, although at least one recent model achieves a several centimeter precision with just several minutes of averaging [Melbourne, 1990]. For this reason we make phase measurements on the carrier itself [Bossler et al., 1980; Remondi, 1985], with an inherent precision of a few millimeters or better. Major remaining error sources include clock biases (in both the satellite and ground receiver, although ground receivers are likely to have larger biases), the atmosphere, including the frequencydispersive ionosphere and the nondispersive troposphere, both of which affect signal velocity and thus our estimate of satellite-receiver distance, uncertainties in the satellite position at the time of signal transmission, and carrier phase cycle ambiguities. The remainder of this paper is devoted to relative (as opposed to point) positioning and associated concepts that enable high-accuracy geodetic measurements with GPS. Relative positioning involves simultaneous observation of a group of satellites by a network of ground receivers. Three-dimensional vectors, termed baselines, are defined between all stations in the network, relative to one or more fixed stations whose positions are known a priori. The combination of simultaneous network observations and the analytical techniques designed to accommodate such data enables us to eliminate or greatly reduce the errors listed above, resulting in the millimeter- to centimeter-level position data we require for most geological and geophysical applications.

\section{Frequency Standards, Time, and Reference Frames}

One key to high-precision geodesy with GPS is simultaneous satellite observations by a number of ground receivers. Simultaneity in this case is defined quite stringently; in 1 millisecond (ms) a station at mid-latitudes moves more than $30 \mathrm{~cm}$ to the east as a result of Earth rotation, a GPS satellite, orbiting at about $3 \mathrm{~km} / \mathrm{s}$, moves 3 $\mathrm{m}$, and a pseudorange signal propagates $300 \mathrm{~km}$. UItimately, we will relate observations at widely separated ground stations to better than a microsecond ( $\mu \mathrm{s})$, although, as we shall see, physical clock synchronization at anything near this level is unnecessary. We nevertheless require a precise time definition and measurement capability, a method for eliminating clock errors, and the ability to relate with great precision the positions of ground receivers anywhere on the Earth to satellites in orbit. The following discussion is summarized from King et al. [1985], Lambeck [1980, 1988], and Leick [1990].

Both GPS satellites and receivers have precise "clocks," i.e., high-frequency, highly stable oscillators. A receiver might employ a quartz oscillator, a mechanical resonator that exploits the frequency-selective properties of the piezoelectric effect, with a fractional frequency stability $\Delta f / f$ of about 1 part in $10^{10}$ per day. Most GPS satellites employ higher-quality rubidium or cesium frequency standards, where atomic resonance phenomena based on the energy difference between two states define the "clock." For example, the cesium clock [Essen and Parry, 1956] is based on the splitting of the ground electronic state of cesium 133, depending on whether the spin of the unpaired valence electron is parallel or antiparallel to the nuclear spin. The transition between these two hyperfine levels has a frequency of $9,192,631,770 \mathrm{~Hz}$ and is the basis for the currently accepted (SI) definition of the second (more on this below).

One measure of clock stability is the Allan (two sample) variance [Allan, 1966]. Following Thompson et al. [1986], if the fractional average frequency deviation $\bar{f}(t)$ from the nominal frequency $f_{0}$ over a time interval $\tau$ is

$$
\bar{f}(t)=[\phi(t+\tau)-\phi(t)] / 2 \pi f_{0} \tau
$$


then the Allan variance $\sigma_{A}^{2}$ is

$$
\sigma_{A}^{2}(\tau)=<[\bar{f}(t+\tau)-\bar{f}(t)]^{2}>/ 2
$$

where the angular brackets denote the infinite time average. The Allan standard deviations $\left(\sqrt{\sigma_{A}^{2}}\right)$ of several common clocks are plotted in Figure 2. Note the long-term stability of cesium clocks, making them attractive for satellite applications, the short-term stability of quartz oscillators, making them adequate for ground receivers, which can be periodically synchronized with satellite signals, and the exceptional stability of hydrogen masers, making them the best choice for ground reference stations.

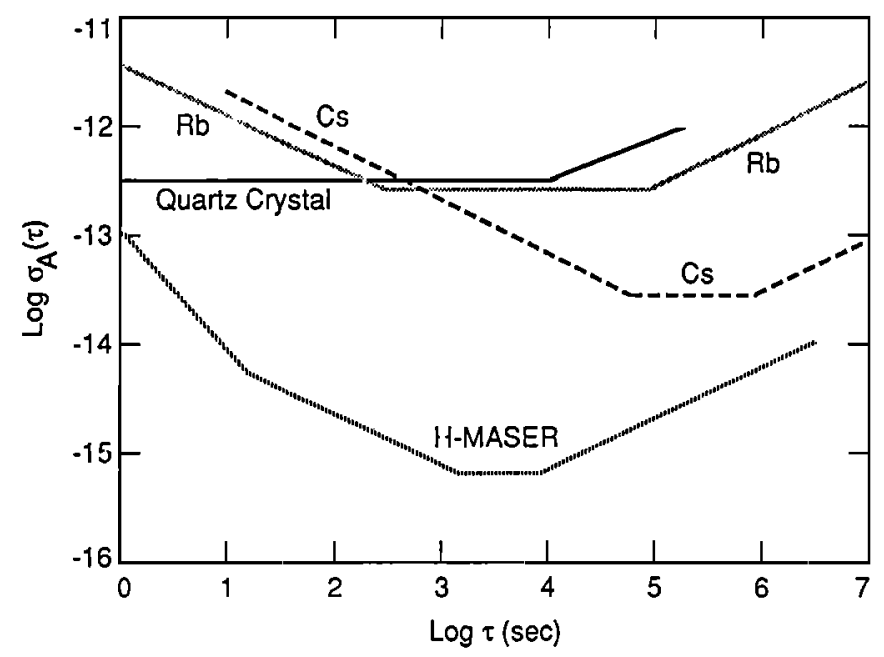

Figure 2. Frequency stability of "best commercial" clocks, measured by the square root of the Allan variance $\left(\sigma_{A}\right.$, equation (7)), as a function of time interval $\tau$. Note the short-term stability of quartz crystal oscillators and the long-term stability of Cs and $\mathrm{H}$ Maser clocks. $\mathrm{Rb}, \mathrm{Cs}$, and $\mathrm{H}$ Maser data from Allan et al. [1989]. Quartz crystal data from Hellwig [1979].

Now that we can measure time, let's define it more precisely. Sidereal "time," the kind many people are familiar with, is based on the Earth's irregular rotation about its axis and is no longer used as a time standard. Ephemeris time (ET) is more regular and exploits the periodic nature of the orbital motions of the Earth, moon, and Sun. Although no longer in use, ET is related to current time standards because the atomic (SI) second is defined equal to the ET second, in turn defined as a fraction of the tropical year 1900. Universal time (UT1) is a form of sidereal time, corrected for some irregularities [Aoki et al., 1982]. Coordinated universal time (UTC) is an internationally accepted standard based on atomic time, roughly synchronized to UT1 by adding "leap seconds" when required. Over the last two decades this addition has averaged about one second per year, reflecting the change of the Earth's rotation rate about its axis with respect to gravitational orbital motion (the basis of the ET, and hence atomic, second). GPS satellites broadcast time signals that are synchronized within $1 \mu \mathrm{s}$ of UTC but are not incre- mented by leap seconds. Thus the difference between GPS time and UTC increases by integral seconds, roughly one per year since 1980 when they were set equal.

We will need to relate the orbital positions of the GPS satellites, computed in a celestial ( inertial) reference frame, to the locations of the ground stations, defined in an Earth-fixed (terrestrial) reference frame. This requires precise knowledge of the Earth's orientation in inertial space. Earth orientation exhibits a rich spectrum of temporal variation. Aside from intrinsic geophysical interest, we need to understand, measure, and correct for these effects in order to relate inertial and terrestrial reference frames. In terms of causes we can distinguish external torques acting on the equatorial bulge of the Earth (forced motion), associated with relative orbital motion of the Earth and moon about the Sun, and free motion, associated with the response of the Earth to internal mass redistribution and corresponding angular momentum exchanges in the Earth system, including the hydrosphere and atmosphere. Effects include changes in spin axis direction with respect to inertial space (precession and nutation), spin axis changes with respect to the Earth's crust (polar motion), and changes in spin rate. Following Sovers and Border [1990], it is convenient to consider the transformation from a terrestrial to inertial reference frame as a series of rotation matrices corresponding to these major effects.

Precession (P) is a large-amplitude $\left(22.5^{\circ}\right)$ slow circular motion of the pole with a period of 26,000 years, caused by lunar-solar attraction on the equatorial bulge. Accurate models are described by Kaplan [1981] and Melbourne et al. [1983, 1985]. Nutations (N) are smaller (maximum amplitude $9 \mathrm{~s}$ of arc), more rapid oscillations superimposed on this precession, with periods from 9 days to 18.6 years. They are excited by external torques, but their response includes a free component. Nutation series are available from the 1980 International Astronomical Union (IAU) theory of nutations [Kaplan, 1981; Seidelman, 1982], based on the theory of Wahr [1981]. Additional annual and shorter-period terms are now known from very long baseline interferometry (VLBI) observations [Herring et al., 1986]. Corrections to the standard precession-nutation model can be incorporated in a perturbation matrix, $\Omega$. Polar motion represents both free and forced responses. In addition to contributions from external torques, possible internal excitation mechanisms include large earthquakes [Slade and Yoder, 1989], aseismic deformation, motions of the atmosphere and hydrosphere, and core-mantle coupling. The dominant period is 14 months (the Chandler wobble), with additional annual and shorter-period motions, causing the pole to wander in a circle less than a few tens of meters in diameter. Pole position is described by two coordinates, $x$ and $y$ (corresponding translation matrices $X$ and $Y$ ), representing rotations about two orthogonal axes lying in the equatorial plane ( $1 \mathrm{~cm}$ of polar motion corresponds to a rotation of about 0.3 millarc seconds (mas). The time- 
varying rotation rate of the Earth, $\mathbf{U}$, and corresponding changes in length of day (LOD), whose annual and shorter-period variations could only be measured after the invention of atomic clocks in the mid-1950s, can be represented by the difference between atomic time (UTC) and UT1. Time series of LOD or UT1-UTC show strong (several milliseconds amplitude) annual and semiannual periods associated with seasonal changes in wind circulation. Shorter-period fluctuations (2 week and lunar monthly) are due to tidal effects, while longer-period "decade fluctuations" may reflect core-mantle interactions (see reviews by Wahr [1988], Lambeck [1988], and Hide and Dickey [1991]). Values for both polar motion (precision 1 mas) and UT1-UTC (precision $0.1 \mathrm{~ms}$ when averaged over a day) come from VLBI observations of quasars [Carter et al., 1985]. Satellite laser ranging (SLR) [Cohen and Smith, 1985] provides corresponding precisions of approximately 2-4 mas and $0.2 \mathrm{~ms}$ averaged over several days [Smith et al., 1985; Tapley et al., 1985]. Lunar laser ranging (LLR) provides roughly comparable data [Dickey et al., 1985]. Sensitivity analyses indicate that systematic errors in GPS orbit and baseline estimates caused by these levels of uncertainty in Earth orientation are negligible [Lichten and Border, 1987; Dixon et al., 1991c].

In summary, spin axis changes with respect to inertial space (precession and nutation) are largely due to external torques (forced motion), can be large in amplitude, and can be modeled quite well. Polar motion is a spin axis change with respect to the Earth's crust, consists of both forced and free motions, is much smaller in amplitude and less easily modeled. UT1-UTC likewise cannot be modeled at present with sufficient accuracy. Both polar motion and UT1-UTC are measured adequately for our purposes by VLBI and SLR.

Tide-related elastic deformations may be important, particularly where high accuracy is desired for widely separated stations (the effects largely cancel for stations close to each other). There are three major effects: the solid Earth tide $\Delta_{\text {sol }}$ (maximum amplitude $\sim 50 \mathrm{~cm}$ ); ocean loading effects in coastal areas, $\Delta_{o c}$ (maximum amplitude $\sim 5 \mathrm{~cm}$ ); and the pole tide $\Delta_{\text {pol }}$, representing elastic response of the Earth to changes in spin axis orientation (maximum amplitude $\sim 1 \mathrm{~cm}$ ). Differential atmospheric loading $\Delta_{\text {atm }}$ [Rabbel and Schuh, 1986] may cause effects at the several millimeter level for large station separations but is not believed to be significant for local or regional GPS networks. This effect may become important with the advent of high-precision global GPS networks.

An Earth-fixed reference frame based on VLBI observations does not precisely define the location of the Earth's center of mass. However, GPS-determined positions are intimately related to GPS satellite orbits, which are sensitive to the center of mass. To account for offsets between the VLBI-based reference frame and the actual center of mass, we define a vector, $r_{c m}$, to be incorporated in the final transformation. SLR defines the center of mass with a precision of about $5 \mathrm{~cm}$ or better [Smith et al., 1985; Tapley et al., 1985]. Colocated VLBI and SLR sites define the center of mass correction for a VLBI reference frame with comparable precision.

The complete transformation from a position vector in a terrestrial reference frame $r_{T}$ to an inertial reference frame $r_{I}$ is given by

$r_{\mathrm{I}}=\boldsymbol{\Omega P N U X Y}\left(\alpha r_{T}+r_{c m}+\Delta_{\text {sol }}+\Delta_{o c}+\Delta_{\mathrm{pol}}+\Delta_{\mathrm{atm}}\right)$

where $\alpha$ is an empirical coordinate frame scaling factor. Note that for local GPS networks, tidal and atmospheric terms can be ignored, and the precision requirements for the other parameters may be less stringent than for large aperture or global networks.

\section{Eliminating Clock Errors: Single and Double Differencing}

A GPS point position is limited to meter-level accuracy by a combination of uncertainties in clocks, orbits, and atmospheric effects. However, for a relative positioning experiment involving a network of ground stations within a specific region, these errors are common mode or nearly so and can be eliminated or greatly reduced in subsequent data processing [Bossler et al., 1980]. Consider two receivers simultaneously observing two satellites. There are four geometric range equations and a maximum of 16 observation equations, since each range could be estimated with both a pseudorange and carrier phase equation, each at two frequencies. For a single-frequency pseudorange measurement, $R$ (equation (1)), denoting satellites $(i, j)$ by superscripts and receivers $(1,2)$ by subscripts, we have the following for satellite $i$ :

$$
\begin{aligned}
& R_{1}^{i}=\rho_{1}^{i}+c\left(\Delta t_{1}-\Delta t^{i}+\Delta t_{p 1}^{i}\right) \\
& R_{2}^{i}=\rho_{2}^{i}+c\left(\Delta t_{2}-\Delta t^{i}+\Delta t_{p 2}^{i}\right)
\end{aligned}
$$

Subtraction gives the single difference observable $R^{\prime}$ :

$$
R^{i^{*}}=\rho^{i^{*}}+c\left(\Delta t_{1}-\Delta t_{2}+\Delta i_{p}^{i^{*}}\right)
$$

where the primed variables denote the differential pseudorange or true range and differential propagation delay, respectively. Satellite clock error $\left(\Delta t^{i}\right)$ is eliminated, and differential propagation delay is considerably smaller than the original delay. Similarly, for satellite $j$,

$$
R^{j}=\rho^{j}+c\left(\Delta t_{1}-\Delta t_{2}+\Delta i_{p}^{j}\right)
$$

Subtraction of equations (11) and (12) gives the double difference observable $R^{\prime \prime}$ :

$$
R^{\prime \prime}=\rho^{\prime \prime}+c\left(\Delta t_{p}^{\prime \prime}\right)
$$

which eliminates receiver clock error and leaves only differential propagation delays as a significant error 
source, which can be calibrated, or solved for, independently (see below). Note that for stations reasonably close to one another (<satellite altitude) the effect of orbit error is also largely common mode. Similar equations can be formed for the carrier phase or beat phase observable [King et al., 1985], which is actually more important for high-precision measurements.

If there are more than two receivers in the ground network, each pseudorange or phase measurement can contribute to more than one differenced observable without adding new information; the observables are correlated, complicating error calculation. While there are methods to deal with these correlations [Beutler et al., 1986; Goad and Mueller, 1988], it may be desirable to operate directly on undifferenced data (sometimes called one-way range or phase), considering clock terms as nuisance parameters to be eliminated by other approaches [e.g., Goad, 1985].

The simple picture of clock error cancellation through simultaneous observations is complicated by SA and receiver clock epoch and sampling offsets. King et al. [1985] provide a complete treatment, but we can illustrate the problem by considering observation in the presence of $\mathrm{SA}$, where the effects are particularly important. SA affects satellite clocks in two ways. First, the satellite clock correction relative to "true" GPS time, normally broadcast in the data message, is corrupted. Fortunately, this has little impact on nonreal time relative positioning applications. Second, clock "dithering" changes the basic $P$ code frequency, resulting in commensurate changes in the derived $L 1$ and $L 2$ signals. Irregular variations of several hertz around the nominal carrier center frequency over periods of a few minutes have been observed with the Block 2 satellites. Since ground measurements are tagged by receive time, but SA effects are common only at the same transmit time, double differencing or analogous schemes to eliminate clock errors can result in incomplete error cancellation. The maximum nonsimultaneity due to SA for receivers at different locations on the Earth is about $20 \mathrm{~ms}$, which, at current levels of SA, induces millimeter-level errors only on the longest baselines. The effects of nonsimultaneity due to receiver clocks are usually negligible with the double-differencing approach, since these clocks can be periodically synchronized to GPS time, for example, with the C/A code. More serious nonsimultaneity (several tenths of seconds) can result simply from different receiver types sampling the signal at arbitrary (and different) times. While there are techniques for dealing with this nonsimultaneity [Feigl et al., 1990a], the simplest approach is to ensure that all receivers are in a network sample at common GPS time. Recent results suggest that with this simple precaution the impact of SA on high-precision geodesy is negligible [Rocken and Meertens, 1990; Feigl et al., 1990a; Rosenblatt et al., 1990]. Additional discussion can be found in the works by Melbourne [1990] and Wu et al. [1990].

\section{The lonosphere}

The GPS signal is affected by propagation through the atmosphere, through changes in velocity, and by ray bending. There are two main regions to consider: the frequency-dispersive ionosphere ( $-50-500 \mathrm{~km}$ altitude) and the nondispersive neutral atmosphere, especially the troposphere ( $-10 \mathrm{~km}$ altitude). Thompson et al. [1986] give a comprehensive review.

Free electrons exist in the ionosphere because solar ultraviolet radiation is absorbed by gaseous molecules in the upper atmosphere, liberating outer shell electrons. The electrons interact strongly with any electromagnetic signal in the frequency range of GPS. Ionospheric effects are proportional to the integrated electron content along the signal path and thus depend on solar activity, the elevation angle of the observation, time of day, and latitude.

The travel time $\tau$ for a group delay measurement can be represented by

$$
\tau=\frac{\rho}{c}+\frac{A}{f^{2}}+\frac{B}{f^{3}}+\cdots
$$

where the term $A / f^{2}$ represents most of the ionospheric delay experienced at frequency $f, A$ is constant, and $B$ is proportional to the average magnetic field strength. If we ignore the third- and higher-order terms, the differential group delay $\Delta \tau$ between observations at $f_{2}$ (the $L 2$ frequency) and $f_{1}$ (the $L 1$ frequency) is [Spilker, 1978]:

$$
\begin{aligned}
\Delta \tau & \approx A\left(\frac{1}{f_{2}^{2}}-\frac{1}{f_{1}^{2}}\right) \\
& =\frac{A}{f_{1}^{2}}\left(\frac{f_{1}^{2}-f_{2}^{2}}{f_{2}^{2}}\right) \\
& =\tau_{1}\left[\left(f_{1} / f_{2}\right)^{2}-1\right]
\end{aligned}
$$

where $\tau_{1}$ is the group delay at $L 1$. Similar equations can be constructed for the carrier phase observable. Dual frequency observations therefore allow elimination of major ionospheric effects (but not third- and higher-order terms) for both pseudorange and carrier phase measurements.

The magnitude of the ionospheric effect can be large. Recalling that $n=c / v$,

$$
n_{\text {ion }} \approx 1-N q_{e}^{2} / 2 \varepsilon_{0} m_{e} \omega^{2}
$$

where $n_{\text {ion }}$ is the ionospheric index of refraction appropriate to the phase velocity of a particular frequency, $N$ is the number density of electrons $\left(\sim 10^{11}-10^{12} / \mathrm{m}^{3}\right), q_{e}$ and $m_{e}$ are the electron charge and mass, respectively, and $\varepsilon_{0}$ is the permittivity. Equivalently, the index of refraction at a given frequency can be estimated from the plasma frequency $f_{p}$ of the ionosphere:

where

$$
n_{\text {ion }} \approx 1-f_{p}^{2} / 2 f^{2}
$$

$$
f_{p}^{2}=N q_{e}^{2} / \pi m_{e}
$$


The plasma frequency is the characteristic vibration frequency for interaction between a plasma (the ionosphere) and an electromagnetic wave; typical ionospheric values are $10-20 \mathrm{MHz}$. Signal attenuation is small for $f \gg$ $f_{p}$, explaining the frequency selection for GPS carriers.

We are more interested in the integrated effect of the index of refraction along the ray path and require the integrated electron density $N_{i}$ (electrons $/ \mathrm{m}^{2}$ ):

$$
N_{i}=\int_{\text {path }} N d z
$$

A useful rule of thumb is that the delay at zenith in equivalent length units, $\mathrm{L}$, is given by $\mathrm{L} \approx 10^{-17} N_{i}$. For zenith observations the magnitude of the time delay associated with the $P$ code observable may be 10 ns or less at night at mid-latitudes $\left(N_{i} \sim 10^{17}\right.$ electrons $\left./ \mathrm{m}^{2}\right)$ and 100 ns or more for daytime observations near the geomagnetic equator and polar regions $\left(N_{i} \sim 10^{18}\right.$ electrons $\left./ \mathrm{m}^{2}\right)$, equivalent to path length variations of several meters to several tens of meters. The delay can also vary as a result of magnetic storms and is sensitive to the 11-year sunspot cycle.

Since the ionosphere is frequency-dispersive, the group velocity $v_{g}$ (associated with the $P$ code pseudorange observable) differs from the phase velocity $v_{\phi}$ (associated with the carrier phase observable); the effects are equal in magnitude but opposite in sign and are referred to as group delay and phase advance. This is suggested by equations (16) and (17), where $n_{\text {ion }}$ is less than 1 for a given frequency, implying that the phase of a wave passing through the ionosphere is advanced relative to a wave traveling in vacuum. Fundamental principles are not violated, because it is $v_{g}$ (the velocity of the code modulation) that determines the speed at which information is carried.

For many applications it is desirable to consider linear combinations of data, to isolate or reduce errors, or to reduce computation time by compressing observations to a single data type. A good example is the ionosphere-free phase observable $L_{c}$ given by [e.g., Blewitt, 1989]:

$$
L_{c}=\left(\frac{f_{1}^{2} L_{1}-f_{2}^{2} L_{2}}{f_{1}^{2}-f_{2}^{2}}\right)
$$

where $L_{1}$ and $L_{2}$ here represent carrier phase ranges at the two frequencies for a particular receiver-satellite pair (i.e., $L_{1}=-c \phi_{1} / f_{1}$ and similarly for $\left.L_{2}\right), L_{c}$ is the ionospherecorrected phase, and the effective wavelength is $\lambda_{c}=c /\left(f_{1}\right.$ $\left.+f_{2}\right) \approx 10.7 \mathrm{~cm}$ (5.4 cm for completely codeless receivers). The shorter effective wavelength of $\lambda_{c}$ implies that resolution of carrier phase cycle ambiguities will be more complicated. For this reason, and also because noise (particularly multipath) is amplified by $L_{c}$, it may be desirable to use single-frequency measurements for very short (a few kilometers or less, depending on ionospheric activity) baselines where ionospheric effects are small or process the two frequencies separately.
It should be clear from the preceding discussion that dual frequency observations are critical to obtaining high-precision geodetic data with GPS. Single-frequency receivers are an economical alternative for certain applications, but if high precision is required, they can only be used on the very short baselines mentioned above, where differential ionospheric effects are small.

The impact of ignoring higher-order terms in the ionospheric delay depends on the electron density in the ionosphere and the dot product of the ray path and the Earth's magnetic field. Several millimeters of range error at low elevation can result. This can be ignored for most applications but may become more important in the future for improved vertical component estimates and tropospheric studies, both of which benefit from lowelevation observations. Dual frequency observations enable estimation of the integrated electron density and in principle could be used to derive models of ionospheric gradients.

\section{The Neutral Atmosphere}

In this section we consider nondispersive atmospheric effects on the GPS signal. These effects are due to the troposphere, tropopause, and mesosphere, but roughly three quarters of the total nondispersive atmospheric delay and most of the variability is associated with the troposphere.

A GPS signal is bent and slowed in its passage through the lower atmosphere. The delay $\tau_{\mathrm{atm}}$ is the difference in travel time between actual signal propagation and the theoretical transit time in vacuum. It is usually expressed as equivalent path length by multiplying by the speed of light and can be defined by the difference of two path integrals [Davis et al., 1985]:

$$
\tau_{\mathrm{atm}}=\int_{\mathrm{atm}} n(s) d s-\int_{\mathrm{vac}} d s
$$

where $n(s)$ is the index of refraction at the point $s$ along the path and $n_{\mathrm{vac}}=1$ is omitted from the second integral. Evaluation of the first integral requires an atmospheric model. All components of the atmosphere contribute to the delay, but it is convenient to consider two components separately. The "dry" delay is associated with molecular constituents of the atmosphere in hydrostatic equilibrium (including $\mathrm{H}_{2} \mathrm{O}$ ), and the "wet" delay is associated with water vapor not in hydrostatic equilibrium. The dry delay is typically $200-230 \mathrm{~cm}$ at zenith (elevation angle, $\theta=$ $90^{\circ}$ ) at altitudes near sea level, while the zenith wet delay might range from 3 to $30 \mathrm{~cm}$. The delay at other elevation angles is larger, increasing approximately as $1 / \sin (\theta)$, but other effects are incorporated in a "mapping function" for greater accuracy, including the finite height of the atmosphere, the vertical distribution of components, Earth curvature, and ray bending [e.g., Black and Eisner, 1984; Lanyi, 1984; Davis et al., 1985]. The total path delay can be written as 


$$
p(\theta)=p_{d}^{0} M_{d}(\theta)+p_{w}^{0} M_{w}(\theta)
$$

where $p^{0}$ refers to the path delay at zenith (hereafter the zenith delay), subscripts $d$ and $w$ refer to dry and wet components, respectively, and $M(\theta)$ is a mapping function, assumed to be azimuthally symmetric.

The dry zenith delay is determined by measurement of surface pressure. If such measurements are not available, standard atmospheric conditions can be assumed, and an initial estimate for the dry zenith delay approximated from the standard pressure $P$ (in bars), based on the surface elevation of the station, $h$, and a scale height $H$, typically 7 km [Tralli et al., 1988]:

$$
P=1.013 e^{-h / H}
$$

Subsequent analysis of the GPS data can improve the initial estimate and reduce the effect of errors in this parameter on the baseline estimates.

The wet zenith delay can be estimated in at least three ways: by measurement of surface temperature and relative humidity coupled with a simple atmospheric model [e.g., Chao, 1974]; with a water vapor radiometer (WVR), an instrument that measures atmospheric blackbody radiation in the microwave region, which is affected by a rotational molecular transition of water vapor near $22.2 \mathrm{GHz}$ [e.g., Janssen, 1985; Robinson, 1988]; and by stochastic estimation techniques without a priori calibration, exploiting the data strength of GPS and the known elevation angle dependence of the wet delay [Tralli et al., 1988; Dixon and Kornreich Wolf, 1990; Dixon et al., 1991a]. Stochastic models make use of the fact that the wet path delay is likely, in a statistical sense, to vary within a limited range over a short time interval. For SM, WVR, or other a priori calibrations, residuals (wet delay corrections) can be estimated along with geodetic parameters of interest. This improves the final result but implies errors in the calibration. Studies have shown that SM calibration by itself can lead to unreliable GPS results [Tralli et al., 1988; Dixon et al., 1991a], confirming earlier studies indicating poor correlation between surface relative humidity and the wet path delay [Reber and Swope, 1972; Elgered, 1982]. K. Hurst et al. (Estimation of GPS baseline errors due to imperfect retrieval of wet atmospheric delays using surface meteorology measurements, submitted to Journal of Geophysical Research, 1991) compare SM and WVR estimates of wet zenith delay and conclude that SM-based estimates would have an rms error proportional to the magnitude of the delay, given by $1.2 \mathrm{~cm}+0.08$ (SMT), where "SMT" is the SM-based zenith delay in centimeters. Tralli et al. [1988] and Tralli and Lichten [1990] suggested that the error in SM calibration can also show considerable time variation. In contrast, corrections to WVR calibration are generally small (1-2 cm in zenith wet delay) and constant or nearly so over several hours, implying that these instruments can give a good indication of temporal variability in the wet zenith delay but may have a small bias that depends on site, season, or data reduction algorithm.

Unfortunately, most WVRs are big and expensive. Fortunately, comparison of GPS baseline estimates involving WVR calibration and stochastic estimation generally indicates no significant differences between the two approaches (for example, Figures 3 and 4), implying that at current precision levels WVR calibration of the wet delay is not required. In fact, it is even possible to lump the wet and dry delays together and estimate them jointly, avoiding all neutral atmosphere calibration (including surface pressure) entirely [Tralli and Lichten, 1990]. This works at current levels of GPS precision because the mapping functions for wet and dry delays are very similar above $10^{\circ}$ or $15^{\circ}$, the typical cutoff angle used with GPS to avoid ground multipath (see below).

In spite of the success of stochastic estimation techniques, for GPS experiments in regions of high wet path delay and high variability (for example, some conditions in the tropics), tropospheric calibration is probably the dominant error source for baselines in the geologically important length range of several tens to several hundred kilometers. Orbital effects dominate at longer baseline lengths, and receiver effects and other errors dominate at shorter baseline lengths. This is discussed in more detail in the section on precision and accuracy. Tropospheric calibration does not appear to be a significant error source in some other regions (for example, typical conditions in the southwestem U.S.) when the wet path delay is low and not particularly variable. Future improvements in the accuracy of GPS, particularly for the vertical component, will depend in part on improved tropospheric calibration and estimation techniques. By analogy with VLBI, direct line of sight calibration with a WVR or other technique may improve GPS baseline estimates when calibration accuracy exceeds 5-10 mm [Herring, 1986; Elgered et al., 1991; Dixon and Kornreich Wolf, 1990]. It has been difficult to deploy high-accuracy WVRs to a majority of sites in a GPS network because of the excessive cost, weight, and power consumption of these sophisticated instruments. Recent developments in the area of monolithic microwave integrated circuit (MMIC) technology have the potential to improve the accuracy and reduce the cost, weight, and power consumption of WVRs, making extensive deployment of these instruments viable. Improved stochastic models are another promising area of research. Current models assume azimuthal symmetry in the wet delay. However, azimuthal asymmetry has been observed [Dixon and Kornreich Wolf, 1990; Rocken et al., 1991]. Estimating two stochastic, orthogonal spatial gradients in addition to the stochastic wet zenith delay should improve precision in the presence of asymmetry and should be feasible with sufficient data strength, presumably available with the enhanced Block 2 constellation and receivers capable of tracking more than four satellites. 


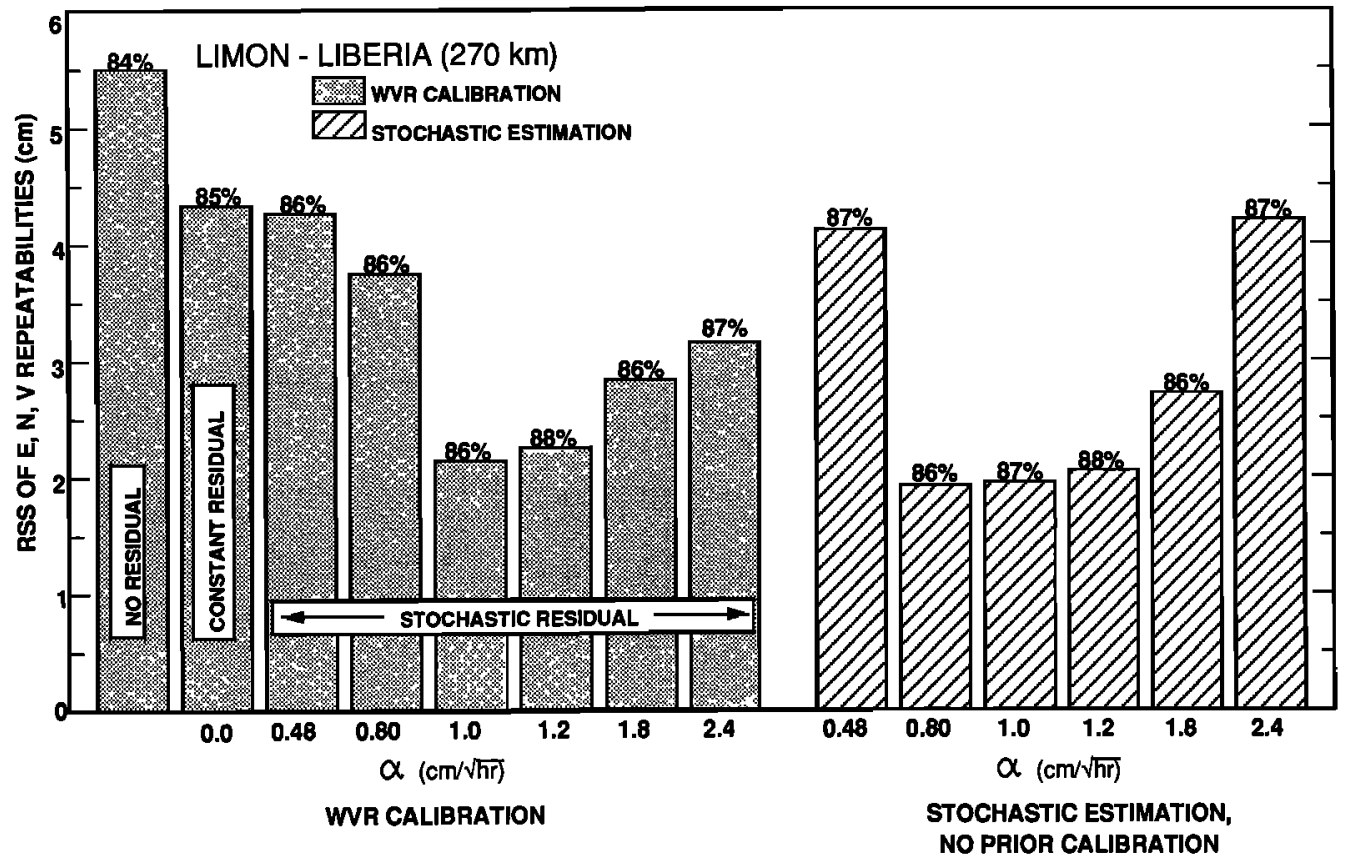

Figure 3. Short-term repeatability for a $270-\mathrm{km}$ baseline in Costa Rica with various treatments of wet path delay (see also Figure 4). Percent symbols above each bar indicate number of biases resolved. With WVR calibration (left side), estimation of a residual delay, either as constant or with a stochastic model specified by the random walk parameter, $\alpha(\mathrm{cm} / \sqrt{\mathrm{hr}})$, improves the calibration, but only to the level reached in the case where no calibration is used (right side) and the entire delay is estimated stochastically [from Dixon and Kornreich Wolf, 1990].

\section{Multipath}

Antennas for almost all GPS receivers are omnidirectional, enabling signals from several satellites to be received simultaneously. Depending on the immediate environment and the gain pattern at low-elevation angles, such antennas can be susceptible to interference ("multipath") from multiple arrivals of the same signal because of reflections from nearby objects. Reflections at the satellite also occur [Young et al., 1985] but can be ignored for most applications. Multipath corrupts the phase and/or pseudo- range observables with systematic, time-dependent sinusoidal signals associated with variable receiversatellite geometry over a pass. The magnitude of multipath is roughly proportional to wavelength, and the effects are considerably larger for $P$ code pseudorange relative to carrier phase. Low-angle observations tend to be most affected, and for this reason a cut-off angle of $10^{\circ}-20^{\circ}$ above the horizon is usually employed. This has the fortunate effect of minimizing errors due to third- and higher-order terms in the ionospheric delay and minimiz-

LIMON - LIBERIA $(270 \mathrm{~km})$
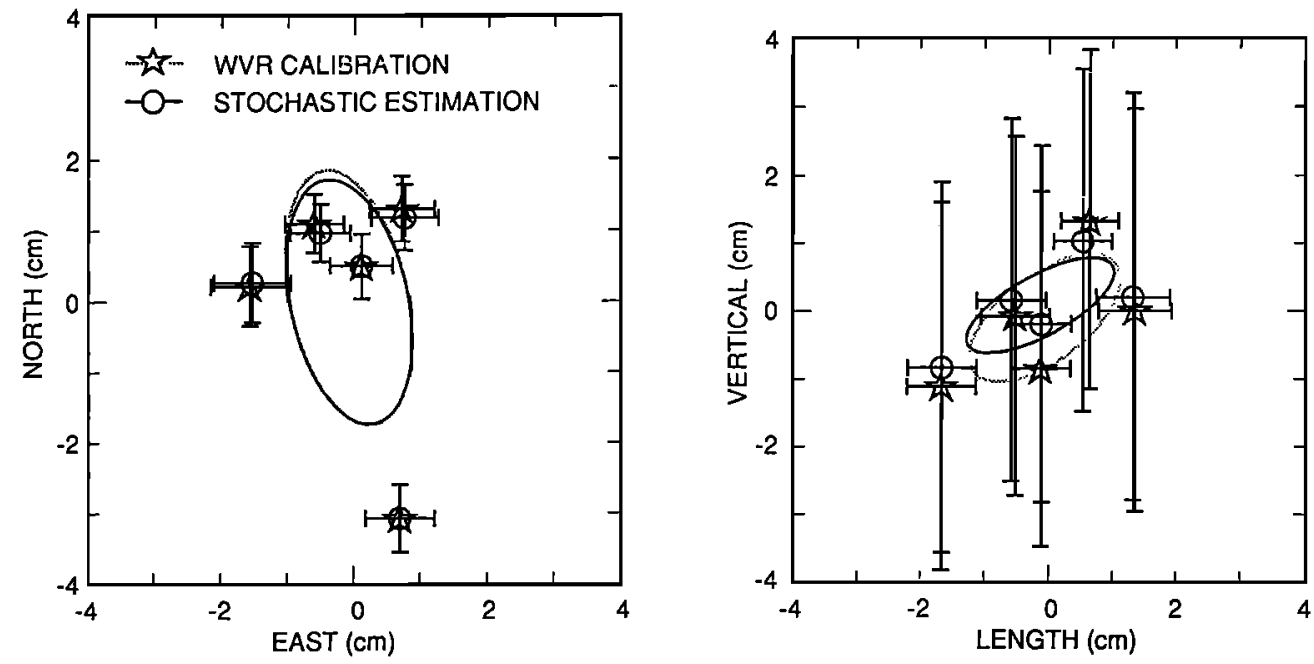

Figure 4. Baseline estimates with and without WVR calibration of the wet path delay for the data shown in Figure 3. Estimates with WVR calibration include a stochastic residual model $(\alpha=1.2 \mathrm{~cm} / \sqrt{\mathrm{hr}})$. Estimates without WVR calibration employ a similar stochastic model for the entire wet delay. Error bars and ellipses are one sigma [from Dixon and Kornreich Wolf, 1990]. 
ing tropospheric calibration errors, including those due to incorrect mapping functions. However, lack of low-angle observations is one of the contributing factors to poor resolution of the vertical component with GPS, so multipath control, as well as improvements in the other areas just mentioned, is highly desirable. Multipath can be minimized through use of antenna backplanes or RF (radio frequency) absorbent material around the base of the antenna, mounting antennas close to the ground (to minimize the effects of ground reflection), and careful site selection, choosing sites well away from planar-reflecting surfaces such as buildings or vehicles.

Since multipath is related mainly to the geometry of nearby objects, and since this geometry is usually constant over several days or longer, the temporal signature of multipath tends to repeat from day to day, retarded by an amount equal to the offset rise time of the satellites ( $4 \mathrm{~min}$ earlier each day). The repetitive nature of multipath in principle can be used to estimate and correct its major effects, though few such studies have been reported. If uncorrected, multipath can be an important source of systematic error, depending on environment, antenna/ backplane design, and length of observing session. Carrier phase multipath tends to have periods shorter than 10-20 min, thus observations over several hours or longer will average out most of the effects.

\section{Orbits}

GPS satellites orbit about three Earth radii above the surface in six orbital planes. For high-precision geodesy it is necessary to know precisely the positions of the satellites at the time of observation, with acceptable error a function of desired baseline precision and station separation; for millimeter-level performance on baselines longer than about $100 \mathrm{~km}$, meter-level precision in the orbit estimates is required.

Satellite orbits can be described by six parameters at some initial epoch and a force model to define subsequent time evolution. The epoch state parameters are three components of position $(x, y, z)$ and three velocities in Cartesian coordinates, or equivalently six Keplerian elements: the semimajor axis (a) and eccentricity (e), describing the size and shape of the elliptical orbit; a parameter describing the position of the satellite within that orbit (for example, $M$, the "mean anomaly" or $f$, the "true anomaly"); and the inclination (i), argument of perigee $(\omega)$, and right ascension of ascending node $(\Omega)$, describing the position and orientation of the orbital plane. In the absence of additional disturbing forces, only the anomaly is time dependent. A simple Keplerian description of an orbit in a plane can be obtained by integrating Newton's laws of motion, giving

$$
r=a\left(1-e^{2}\right) /[1+e \cos (f)]
$$

where $r$ is radial distance from the center of the Earth [Kaula, 1966]. For near-circular orbits like GPS, $e \sim 0$. The nature of the perturbing forces on the satellites and the requirement for high-accuracy means that this model is not adequate for our purposes. Rizos and Stolz [1985] summarize major accelerations on the GPS satellites. Atmospheric drag is negligible at these altitudes, and the Earth's nonsymmetric gravity field, while significant, is adequately described with current models; the more poorly determined short wavelength components, related mainly to lithospheric structures in poorly surveyed parts of the Earth, have little effect on the high-altitude GPS satellites. Additional large perturbations are the gravitational effects of the moon and Sun, which can be accurately determined, and solar radiation pressure.

One approach to the orbit problem is to begin with an estimate of the six orbital components at some initial time and numerically integrate the equations of motion using accurate models for various pertubing forces and resulting accelerations, predicting location and velocity of the satellites at later epochs; ground tracking data can be used in a least squares adjustment to improve the initial position/velocity estimates, the model parameters, and the subsequent position/velocity estimates. The length of the orbit arc over which the equations are integrated may be only a few hours ("short arc") in which case the force models can be relatively simple, or may extend to several weeks ("multiday" or "long arc"), requiring sophisticated force models. Solar radiation pressure produces a large and somewhat unpredictable perturbing force because of the large cross-section area of the solar panels, complex satellite geometry, and variable satellite albedo, resulting in accelerations of order $10^{-7} \mathrm{~m} / \mathrm{s}^{2}$ and perturbing the orbits many meters in just a few hours. Although a solar radiation pressure model for the GPS satellites accounts for albedo and geometry, large residuals have been observed [Fleigel et al., 1985]. It is therefore common to solve for at least one additional acceleration parameter in the estimation process, representing departures from the ideal model. In a spacecraft-centered coordinate system where $z$ points to the center of the Earth and $y$ is along the solar panel support beam and normal to the spacecraft-Sun direction, unmodeled accelerations are often observed in the $y$ direction ("y-bias"), perhaps related to thermal radiation or misaligned solar panels [Fleigel et al., 1985; Schutz et al., 1990]. Residuals tend to be largest during the 2-month eclipse season, when the spacecraft periodically enter the Earth's shadow [Schutz et al., 1990]. Lichten and Border [1987] adjusted solar pressure coefficients in all three components for data arcs up to 1 week, using constant corrections to the nominal model. Lichten and Bertiger [1989] used three component stochastic corrections to the nominal model for data arcs longer than 1 week.

Information on satellite trajectories during the period of the experiment may come from the broadcast ephemeris in the GPS signal, or additional GPS data taken simultaneously from sites whose positions are well known from independent measurements such as VLBI or SLR. The resulting tracking data allow generation of accurate 
ephemerides (satellite locations as a function of time) and define the reference frame for the experiment. The subnetwork of known ground stations is called a fiducial network. Uncertainties in fiducial site location, or in the ground surveys connecting the phase centers of the large VLBI antennas to the ground mark used by the GPS antenna, or low-quality GPS data at one or more of these sites during an experiment, are a major source of systematic error in GPS geodesy.

That part of the error in a baseline estimate $\sigma_{b l}$ due to orbit error $\sigma_{\text {orb }}$ can be estimated from the rule of thumb $\sigma_{b l}$ $\approx \beta \sigma_{\text {orb }} L / h$, where $L$ is baseline length, $h$ is satellite altitude, $\sim 20,000 \mathrm{~km}$, and $\beta$ is a geometric factor $(\sim 0.2)$ [Lichten, 1990a]. Since the error in the broadcast ephemeris can be $50-100 \mathrm{~m}$ with selective availability, we require a robust fiducial network for any experiment with baselines longer than about $50-100 \mathrm{~km}$ if we desire centimeter level or better accuracy. The nature of the required network depends on the experiment. In general, at least three stations are required, with adequate north-south and east-west extent. Stations too close to each other provide redundant information and may not contribute greatly to network strength. On the other hand, mutual satellite visibility is desirable for cancellation of clock errors, one or two redundant stations protect against data outages, and some proximal stations can facilitate cycle ambiguity resolution. The optimum number and geometry of stations may be difficult to predict. A covariance analysis, which estimates uncertainties in baseline vectors from certain assumptions about the data and its errors, can be useful in testing trial fiducial networks [Dixon et al., 1985; Freymueller and Golombek, 1988].

In some cases, covariance analyses may suggest the need for fiducial sites in areas where VLBI or SLR sites are not available, or where the necessary ground tie data are not available. It may still be advantageous to deploy a receiver to such a location to provide additional geometric strength to the tracking data. Kornreich Wolf et al. [1990] used data from the "CASA" experiment to show that adding two tracking sites in the southwest Pacific and two in Europe to a fiducial network consisting of three stations in the U.S. improved the repeatability of the horizontal components of long $(>400 \mathrm{~km})$ baseline estimates in Central America and northern South America (Figure 5), even though the actual location of the new stations was uncertain at the $10-20 \mathrm{~cm}$ level. The position of such tracking sites is estimated in the data analysis to avoid introducing systematic error.

In spite of our best efforts to establish a robust fiducial network we may find that logistical limitations or data outages limit the quality of the resulting observations. An important clue to the cause of low-quality data can be found by plotting repeatability (day-to-day scatter) of baseline estimates as a function of baseline length; a high dependence on length $\left(\geq 2-3\right.$ parts in $10^{8}$ for baselines longer than 100-200 km) suggests the possible influence of orbit errors (Figures 5 and 6). It may then be ad- vantageous to employ a multiday arc analysis, instead of the single day, short arc approach. The advantages of multiday arc analysis are described by Lichten and Border [1987]. With observations over more than one revolution, orbital periods are more accurately determined and the
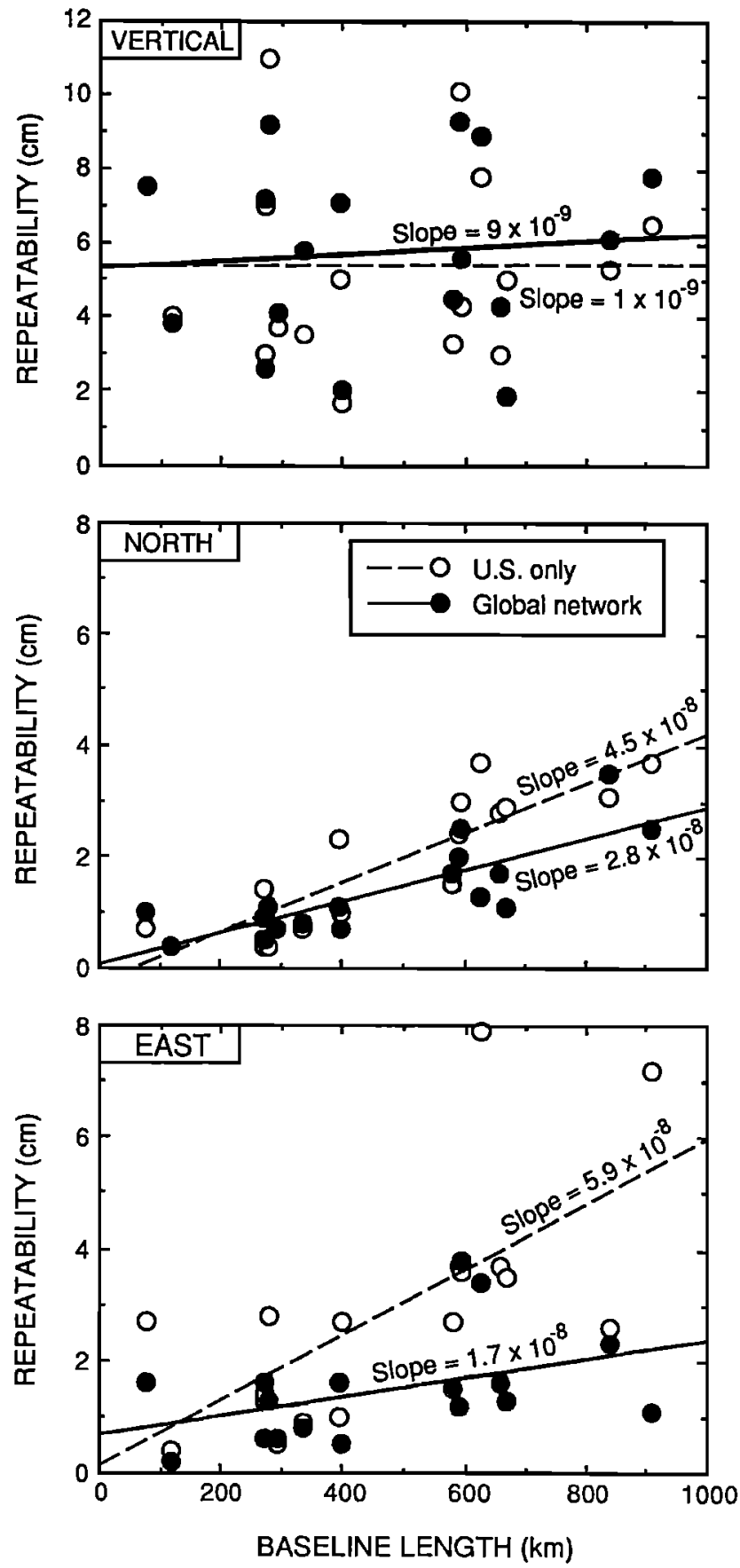

Figure 5. Improvement in short-term (3-8 days) repeatabillty (equation (30)) for seventeen baselines in Central and northem South America as a function of fiducial network configuration. Single-day orbital arcs are used. "U.S. only" indicates three VLBI sites in the U.S. "Global" indicates this network supplemented by two European and two southwest Pacific stations. Best fit straight lines are shown (modified from Kornreich Wolf et al., [1990]. 
positions of the orbital nodes better defined, with corresponding improvement in the ability to resolve carrier phase cycle ambiguities throughout the network and the precision and accuracy of all components of the baseline vector. Figure 6 shows the repeatability of a set of baseline vectors from the northern Caribbean before and after a multiday arc analysis [Dixon et al., 1991a]. Geodetic estimates were improved by factors of 2 or more with the longer arcs for baselines longer than about $500 \mathrm{~km}$. Single-day arcs and the existing three-four station fiducial network were apparently adequate to minimize the contribution of orbit error for shorter baselines.

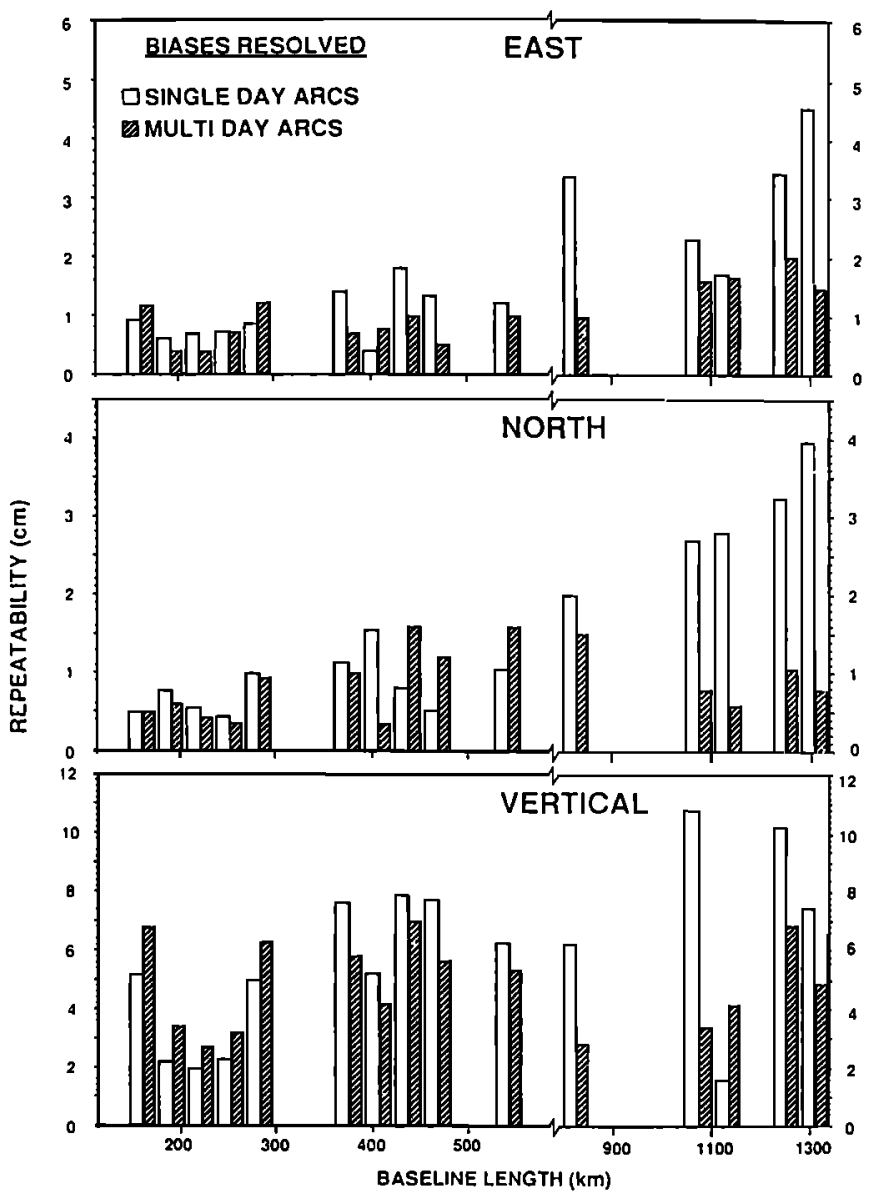

Figure 6. Histograms of short-term repeatability (equation (30)) for 15 baselines in the northern Caribbean for single day and multiday orbital arc analysis. Carrier phase cycle ambiguities ("biases") are resolved for each technique to the extent possible. Improvement in the longer $(>500 \mathrm{~km})$ baselines with multiday arcs results in part from the increased ability to resolve the biases [from Dixon et al., 1991a].

The burden of establishing adequate fiducial networks for regional GPS experiments will be considerably reduced in the next few years as various national and international agencies establish global tracking networks. The Cooperative International GPS Network has been in operation with six or more stations since 1989, administered in the U.S. by the National Geodetic Survey. NASA will sponsor a six-station GPS global tracking network, expected to be operational in 1991, for tracking the U.S.-French TOPEXPoseidon satellite, a low Earth orbiter for oceanographic research to be launched in 1992. Neilan et al. [1990] give a current summary of international tracking networks.

\section{Reducing the Data}

Consider a hypothetical GPS experiment involving 5 days of observations at 10 ground stations, with the positions of three fiducial sites well known. Each station might observe six satellites for an average of 5 hours each day (the total time span of observations might be 8 hours or more, but not all satellites are visible simultaneously). Pseudorange and/or phase data are collected continuously, but let's consider phase data averaged at 1-min intervals. The total number of "data points" collected in 1 day is then 10 stations $\times 6$ satellites $\times 5$ hours $\times 60$ points per hour $=$ 18,000 . From this large data set we might estimate three position components for each unknown ground station (21), six components (three position, three velocity at the initial epoch) for each satellite (36), a $y$ bias term for each satellite (6), perhaps one troposphere parameter for each ground station (10) (additional terms are required for a stochastic model), and clock terms every minute for all satellites (1800) and nine ground stations (2700), since one station acts as a reference clock, giving a total of 4573 unknowns. The system is overdetermined, with data points greatly exceeding unknowns. Since the data are noisy, we cannot formulate simultaneous equations to solve directly for the desired parameters. The challenge is to find, in a least squares sense, the best estimate of the parameters from available data, using where possible additional information such as tropospheric calibration and orbit models. There are numerous approaches to GPS data analysis, and I have tried to generalize the following discussion. However, some details are specific to analytical approaches familiar to me, and I apologize for this obvious bias.

The time evolution of pseudorange or phase observables (e.g., equation (3)) is largely a function of observation geometry. Given prior knowledge of the approximate ground station positions in a terrestrial reference frame, satellite orbits (from the broadcast ephemeris), Earth orientation, and initial estimates of atmospheric delay, it is possible to generate fairly accurate models for the observables. With these models, and knowledge of how the observable $o b$ would change given a small change in a parameter, $x$, (given by the partial derivative, $\delta(o b) / \delta x$ ), least squares or Kalman filter techniques can be applied to the observables to improve estimates of $x$. Following King et al. [1985], given an observable model $c$, an observation $l$, and an observation error $v$ (all functions of time),

$$
c=l+v
$$

This can be linearized by expanding the left-hand side in a Taylor series and dropping second- and higher-order terms, expressing $c$ as a vector of initial estimates of the observ- 
ables, $c_{a}$, based on a priori values of $x, x_{a}$, plus incremental corrections, $[\delta(o b) / \delta x] d x$, to be constrained by the data and its errors. The linearized observation equation in matrix form is

$$
c_{a}+\mathbf{A} d x=l+v
$$

where $A$ (the design matrix) is the matrix of partial derivatives, $d x$ is a vector of small corrections to $x_{a}$, and $v$ is now the vector of postfit residuals. The quantity minimized is the weighted sum of squared residuals, given by $v^{T} \mathbf{W} v$, where $W$, the weight matrix, is the inverse of $P$, the covariance matrix. The solution is

$$
\hat{x}=x_{a}+d x
$$

where $\hat{x}$ is the best estimate of $x, d x=\mathbf{N}^{-1} \mathbf{A}^{T} \mathbf{W}\left(l-c_{a}\right)$, and $\mathbf{N}$ is the normal equation matrix given by $A^{T} \mathbf{W A}$. This approach may be applied to both differenced and undifferenced data. It is also possible to transform undifferenced data to derived parameters containing no clock terms through orthogonalization methods without forming normal equations [Lawson and Hanson, 1974]. Additional discussion of GPS data analysis approaches can be found in works by Goad [1985], Bock et al., [1985a, b], Lindler and Wells [1985], Bock et al. [1986], Beutler et al. [1986, 1987], Schaffrin and Graffarend [1986], and Leick [1990].

Most of the data discussed in this report were processed with the GPS Inferred Positioning System (GIPSY) software developed at the Jet Propulsion Laboratory. GIPSY processes undifferenced data using a modified Kalman filter, described by Lichten [1990b]. Briefly, a conventional Kalman filter updates measurements from one observation epoch to the next on the basis of the covariance matrix $\mathbf{P}$. Factorization of $\mathbf{P}$ into upper triangular (U) and diagonal (D) matrices $\left(\mathbf{P}=\mathbf{U D U}^{T}\right)$ improves accuracy and computational efficiency [Thornton and Bierman, 1980] and is the basis for the parameter estimation. The formulation of the linearized least squares observation equation differs slightly from (26), partitioning parameters into a satellite state vector $(x)$, a vector of constant parameters $(y)$, a vector of process noise parameters $(p)$ and $v$, defined here as a zero mean white noise vector:

$$
\mathbf{Z}=\mathbf{A}_{\mathbf{x}} \mathbf{X}+\mathbf{A}_{p} \mathbf{P}+\mathbf{A}_{\mathbf{y}} \mathbf{Y}+\boldsymbol{V}
$$

where $\mathbf{z}$ is the difference between an observation and its model value. Parameters modeled as process noise include clocks (modeled as white noise) and wet tropospheric path delays (modeled as random walks). Measurements are grouped into finite time intervals or batches (typically one to several minutes) and processed sequentially, leading to updated parameter estimates and covariances after each batch. After filtering all data a smoothing algorithm can be applied that works backward in time to update the estimates and covariances, for example, allowing investigation of the time-varying behavior of the troposphere [e.g., Dixon and Kornreich Wolf, 1990].

We saw from equations (2) and (3) that the initial phase measurement upon acquisition of the carrier signal is biased by an unknown number of cycles. Assuming a receiver maintains lock on the signal, the range change between the receiver and satellite can be determined, and the initial range (the cycle ambiguity) can be estimated along with the geodetic parameters of interest. However, this degrades the accuracy of horizontal baseline components relative to the case where the cycle ambiguity is known or can be fixed to the correct value. Techniques to resolve the ambiguity rely on the fact that, given enough data, the range bias can be estimated to better than half a carrier wavelength (cycle), after which the bias is fixed to the nearest integer value. Orbits, ionospheric effects, tropospheric effects (Figure 7), multipath, and other error sources can corrupt GPS signals such that errors become significant relative to one half wavelength, affecting our ability to fix ambiguities to the correct value. It is common practice to resolve the ambiguities first on shorter $(<100$ $\mathrm{km}$ ) baselines where the effect of most of these errors is reduced [Bock et al., 1985b; Abbot and Counselman, 1987; Dong and Bock, 1989; Blewitt, 1989]. The method used for most of the data presented here also exploits the fact that

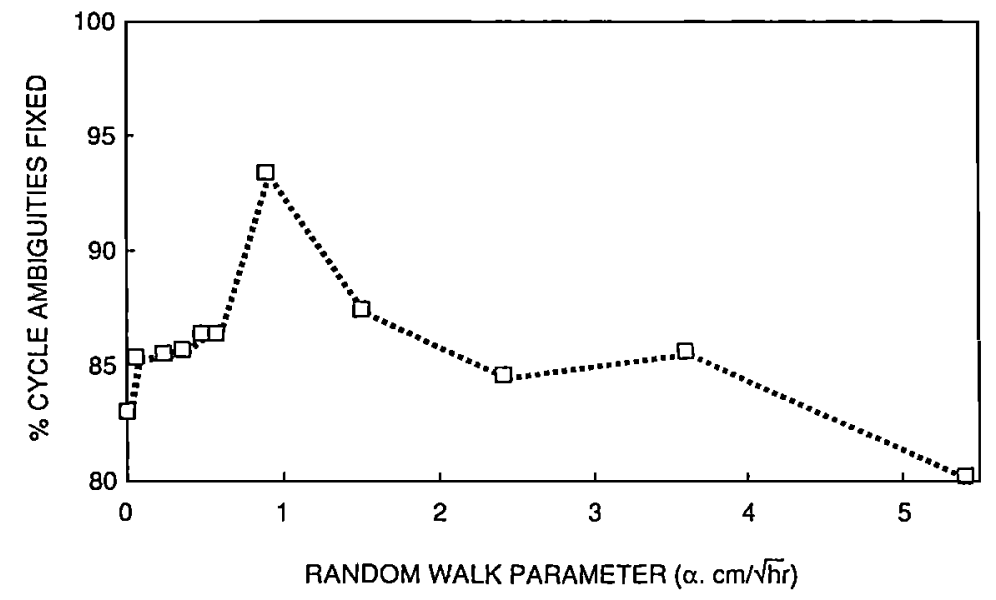

Figure 7. Percentage of carrier phase cycle ambiguities (biases) resolved as a function of the stochastic (random walk) model used to characterize the wet zenith path delay for a GPS network in the northern Caribbean. Optimum model has random walk parameter $\alpha=1.0 \mathrm{~cm} / \sqrt{\mathrm{hr}}$ (compare to Figure 3) [from Dixon et al., 1991a]. 
the ionospheric group delay of the $P$ code modulation is the same magnitude (though opposite sign) as the phase delay, provided the correct number of cycles is associated with the phase measurement [Melbourne, 1985; Wubbena. 1985]. Blewitt [1989] gives a complete discussion.

Another "bootstrap" technique that assists bias resolution involves the "wide-lane" ambiguity. A linear combination of observables, the wide-lane phase $L_{w}$, defined as

$$
\begin{aligned}
L_{w} & =-\left(\phi_{1}-\phi_{2}\right) \lambda_{w} \\
& \left.=\left(f_{1} L_{1}-f_{2} L_{2}\right) / f_{1}-f_{2}\right)
\end{aligned}
$$

is useful because the wide-lane wavelength, $\lambda_{w}=c /\left(f_{1}-f_{2}\right)$ $\approx 86 \mathrm{~cm}$, is more easily resolved than the "narrow-lane" $\left(\lambda_{1}, \lambda_{2}\right)$ ambiguities (compare equations (20) and (29) and definitions of $\lambda_{w}$ and $\left.\lambda_{c}\right)$. Subsequent techniques can be applied iteratively to resolve the narrow-lane ambiguities $\left(\lambda_{c} \approx 10.7 \mathrm{~cm}\right)$ as discussed above. With $P$ code receivers the wide-lane ambiguity may be resolvable even if the $P$ code data are relatively noisy ( $P$ code error averaged over several hours just needs to be less than $\lambda_{w} / 2$, i.e., $<43 \mathrm{~cm}$ ). Codeless receivers have an effective wide-lane wavelength of only $43 \mathrm{~cm}$, thus the wide-lane ambiguity is more difficult to resolve.

Most high-precision relative positioning experiments with GPS are "static," in the sense that a given receiver observes in one position for many hours or days to obtain data strength adequate to resolve the cycle ambiguities and reduce other errors. However, over short $(<30 \mathrm{~km})$ baselines, it is also possible to operate in a "kinematic" mode, with a receiver maintaining lock on the satellite signals and moving from station to station, continuously recording data. As long as lock is maintained, only one cycle ambiguity per receiver-satellite pair needs to be resolved, regardless of the number of stations covered by the roving receiver. The technique has obvious advantages for rapid survey of large numbers of points. A variation of this approach, "rapid static" surveying, does not require continuous signal lock, exploiting high-quality $P$ code data or prior data to resolve the ambiguities directly [Blewitt et al., 1989].

The assumption of linearity (equations (26) and (28)) means that the initial model for the observables must be sufficiently accurate that second- and higher-order terms can be neglected. Accuracy can be estimated after the fact by inspecting the solution offset from the assigned a priori value. The solutions should be iterated unless the satellite epoch states are accurate to about $\mathbf{1 ~ k m}$, and initial ground positions accurate to several hundred meters [Lichten, $1990 b]$.

\section{Experiment Design}

On the basis of the preceding discussion we can summarize the important aspects of a successful highprecision GPS geodetic experiment. Additional discussions can be found in works by King and Blewitt [1990], Dixon et al. [1991a, b], Blewitt [1990], and Bevis [1991]. From our discussion of the precision of carrier phase measurements and ionospheric effects it should be clear that phase-tracking, dual-frequency receivers are critical. $P$ code pseudorange capability is useful but not critical for editing cycle slips, high-precision clock synchronization, and carrier phase cycle ambiguity resolution. If $P$ code is not present, $C / A$ code pseudorange can provide clock synchronization to the microsecond level or better. The ability to track six or more satellites is helpful. If possible, common receivers and antennas should be used throughout the network to ensure common time tagging in the presence of SA and reduce the effect of antenna phase center variation [Rocken and Meertens, 1989].

In addition to adequately sampling the geophysical phenomenon of interest, two other aspects of network design are important. First, if baselines longer than about 50-100 km are to be determined with high accuracy, an adequate (three or more station) fiducial network is important. A covariance analysis can be performed to determine optimum configuration. Variations in the number, geometry, and quality of fiducial stations between experiments can lead to large, spurious variations in geodetic position estimates [Larson, 1990a]. Second, the region of interest must be covered with a sufficiently dense network of stations to ensure resolution of carrier phase cycle ambiguities. If a large region must be covered with a sparse network, at least 2-3 baselines should be shorter than $100 \mathrm{~km}$. If necessary, the network can be covered in two or more observing sessions with a few common stations [e.g., Kellogg et al., 1989].

With the satellite constellation available up until 1990 , high-precision results have been achieved in several regions with 3-5 days or more of observation, assuming at least 7-8 hours of observation per day [e.g., Tralli and Dixon, 1988; Dong and Bock, 1989; Dixon et al., 1990; Kornreich Wolf et al., 1990; Kellogg et al., 1990]. Observing for two or more days allows a check of data quality by treating each day independently in the subsequent analysis and comparing results and reduces the effect of random error by averaging. Another advantage of several days of observation is that multiday orbital arc analysis is possible.

The duration of observations in a given day depends on experiment location and satellite constellation geometry, as well as receiver design, multipath environment, and logistical considerations. Experience with the Block 1 constellation using mainly four-channel receivers allows some generalizations. King and Blewitt [1990] suggest that observing for 6.5 hours or less per day may degrade results. Freymueller and Golombek [1988] compared 7and 9-hour observations for a GPS experiment in northern South America with covariance analyses and suggested improvement with the longer observing time. Davis et al. [1989] reported that carrier phase cycle ambiguities could not be resolved reliably even on short $(30-50 \mathrm{~km})$ baselines with short ( 4.5 hour or less) observing sessions. 
Optimum observing times may decrease once the Block 2 constellation approaches operational capability, assuming receivers are capable of tracking more than four satellites and depending on SA effects. High-quality $P$ code data, if available, could also shorten observing times by providing constraints on carrier phase cycle ambiguities.

Finally, site selection and site integrity are important. Sites should have adequate sky visibility and be located away from buildings or other reflecting surfaces that introduce multipath. Instability of the ground monument may be a substantial source of noise in a long-term GPS experiment, introducing spurious position shifts unrelated to tectonic signals, and is an important and often overlooked aspect of GPS experiment design. Monuments not emplaced in bedrock are most susceptible and may require special design [Wyatt, 1989].

\section{PRECISION AND ACCURACY}

The precision and accuracy achievable in a given GPS experiment have improved greatly in the last decade with better experiment design, receiver hardware, and analytical techniques, and further improvements are likely. I will address mainly "best achievable" accuracies; in some cases an application may have less stringent accuracy requirements, and considerable savings in field operation and data analysis costs may be possible by accepting slightly reduced accuracy.

Both precision and accuracy of GPS baseline estimates depend strongly on whether or not the carrier phase cycle ambiguities have been resolved [Blewitt, 1989, Dong and Bock, 1989]. Bias fixing improves the precision and accuracy of GPS horizontal baseline component estimates by factors of 2-3; the vertical component is usually unaffected (Figure 8). The ability to resolve the biases is a good indication that certain errors have been reduced to some threshold value; in general, it is difficult to resolve the biases with lower quality data. For consistency, most of the examples discussed in this section will therefore involve bias-fixed data.

As with most physical measurements, it is useful to distinguish random errors, the effects of which can be reduced by averaging more data, and systematic errors. The major random error source in GPS is the noise of the phase observable. Systematic errors are of two types. The first is due to errors in the input (nonestimated) parameters, for example, fiducial site location, Earth orientation, and

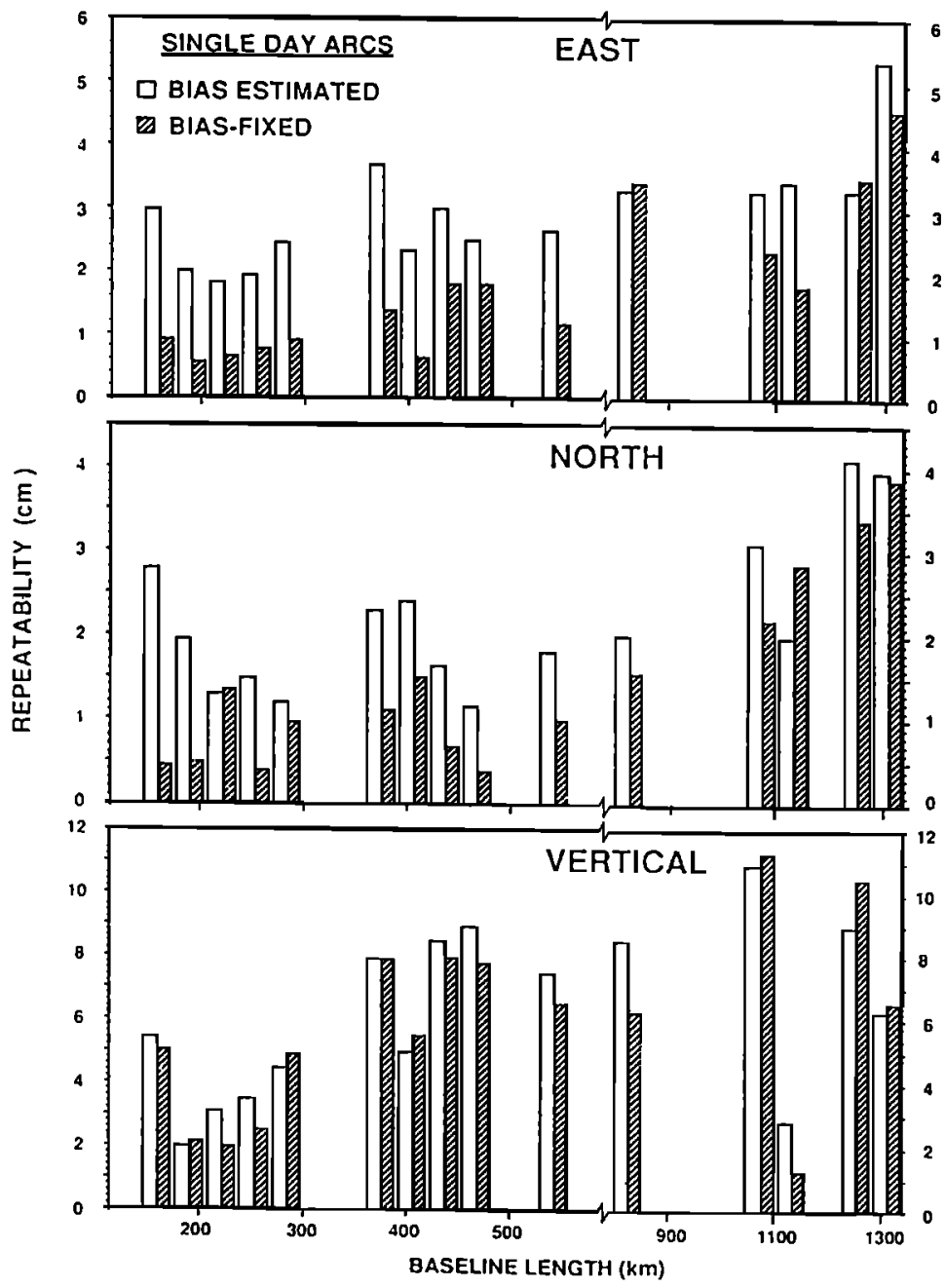

Figure 8. Histograms of short-term repeatability (equation (30)) for 15 baselines in the northem Caribbean before and after resolution of carrier phase cycle ambiguities (bias fixing). Note improvement in horizontal components for baselines shorter than about $500 \mathrm{~km}$. Biases could not be resolved on the longer baselines with single-day orbital arcs (compare to Figure 6) [from Dixon et al., 1991a]. 
geocenter coordinates. Sensitivity analyses suggest that only fiducial site location error is significant [Lichten and Border, 1987; Dixon et al., 1991c]. The second type of systematic error is associated with models constraining the estimated parameters. For example, errors in models of the Earth's gravity field and the solar flux cause errors in the subsequent orbit and baseline estimates. Sensitivity analyses suggest that these orbit-related errors are negligible. Other systematic errors of this type result from assumptions and models concerning the atmosphere, for example, ignoring third- and higher-order terms of the ionospheric correction, use of incorrect mapping functions (both dry and wet) especially at low elevation angles, and the assumption of azimuthal symmetry in this dynamic and nonhydrostatic medium. Although it is difficult at present to quantify these systematic errors, the data presented below suggest some bounds.

One measure of the uncertainty of a GPS vector baseline estimate is the formal error, based on propagation of random data noise through the estimation process. Formal error based only on data noise, even if scaled to reflect postfit residuals, underestimates true error as it does not account for all random errors nor most systematic errors. However, formal error does allow us to propagate uncertainties and describe correlations in a statistically rigorous manner.

Assuming that each day of data is treated independently, the scatter of daily solutions for a GPS experiment spanning several days (the "short-term" repeatability) is another measure of GPS performance and is one indicator of precision. The repeatability $R$ of a vector baseline component (east, north, or vertical) is the root-mean-square (rms) scatter about the weighted mean:

$$
R=\left(\frac{\frac{n}{n-1} \sum_{i=1}^{n} \frac{\left(c_{i}-\langle c\rangle\right)^{2}}{\sigma_{i}^{2}}}{\sum_{i=1}^{n} \frac{1}{\sigma_{i}^{2}}}\right)^{1 / 2}
$$

where $n$ is the number of days, $c$ is the estimate of the component on day $i,\langle c\rangle$ is the weighted average of the component estimate over all days, and $\sigma_{i}$ is the formal error. Short-term repeatability has been used to assess the relative merits of various experimental and analytical approaches [Tralli and Dixon, 1988; Dong and Bock, 1989; Blewitt, 1989; Dixon et al., 1991a]. Note that if just a few days of data are available for a small number of baselines, the statistical significance of the observed scatter may be limited. Also, short-term repeatability will not reveal a slowly varying systematic error, for example, due to propagation delay, multipath, or fiducial network inconsistencies, which might be constant over several days. On the other hand, since random errors are reduced with repeated measurements, repeatability might actually be pessimistic. With more data now available it is possible to use another indicator of precision, the "long-term" repeatability, the scatter of a series of GPS measurements taken over months or years, which will show the effects of slowly varying errors. For stations not separated by an active fault, no motion is assumed on the baseline, and (30) can be used. For stations separated by an active fault we can assume steady motion and look at the scatter of points about a best fit straight line on a plot of position versus time [Davis et al., 1989; Larson, 1990b]. An important question is the relation between short- and long-term repeatability.

The overall precision of an individual GPS baseline estimate will depend on several factors, whose contribution to the total will be the square root of the summed squares (rss) of the individual error terms. Some of these errors will be constant, while others will depend on baseline length. A simple formalism first proposed by Savage and Prescott [1973] to describe the precision of Geodolite distance measuring equipment is

$$
\sigma=\left(a^{2}+b^{2} L^{2}\right)^{1 / 2}
$$

where $\sigma$ is the standard deviation, $L$ is baseline length, and $a$ and $b$ are the constant and length-dependent sources of error, respectively, with $a=3 \mathrm{~mm}$ and $b=2 \times 10^{-7}$. For comparison, the length component of a VLBI position vector has $a=5.4 \mathrm{~mm}$ and $b=2.4 \times 10^{-9}$ [Clark et al., 1989]. Equation (31) has also been used to describe the precision of GPS measurements as a function of baseline length [Dixon et al., 1990; Hager et al., 1991]. As we shall see, this simple error model has weaknesses when applied to GPS, but is adequate to address some basic questions, in particular the relation between short- and long-term repeatability. Best fitting curves (31) through some short-term repeatability data for GPS experiments in the southwestern U.S. [Dixon et al., 1990] and the northern Caribbean [Dixon et al., 1991a] give, for horizontal (north and east) components, $a \approx 3-8 \mathrm{~mm}$, and $b \approx 1-2 \times 10^{-8}$ (Figure 9). The east component is determined more poorly than the north, because the satellite ground tracks were oriented mainly north-south, giving large range-change "signals" in the north-south direction, and smaller signals in the east-west direction. The difference is reduced with bias-fixed data. The vertical component is the most poorly determined with GPS, reflecting the geometric limitation that satellites are observed in the upper hemisphere only, and higher sensitivity (about a factor of 3 relative horizontal components) to errors in tropospheric calibration [Herring, 1986]. Typical values for vertical repeatability are in the range $20-60 \mathrm{~mm}$, with the higher values indicative of high and variable wet tropospheric path delay (Figure 9). Vertical repeatability is not a strong function of baseline length and thus is not well fit by equation (31).

The question of short- versus long-term repeatability can be partially answered by comparing these data to data from later experiments. Figure 10 shows results from seven GPS experiments conducted over a 3-year period between Mojave (on the North American plate) and Vandenberg 

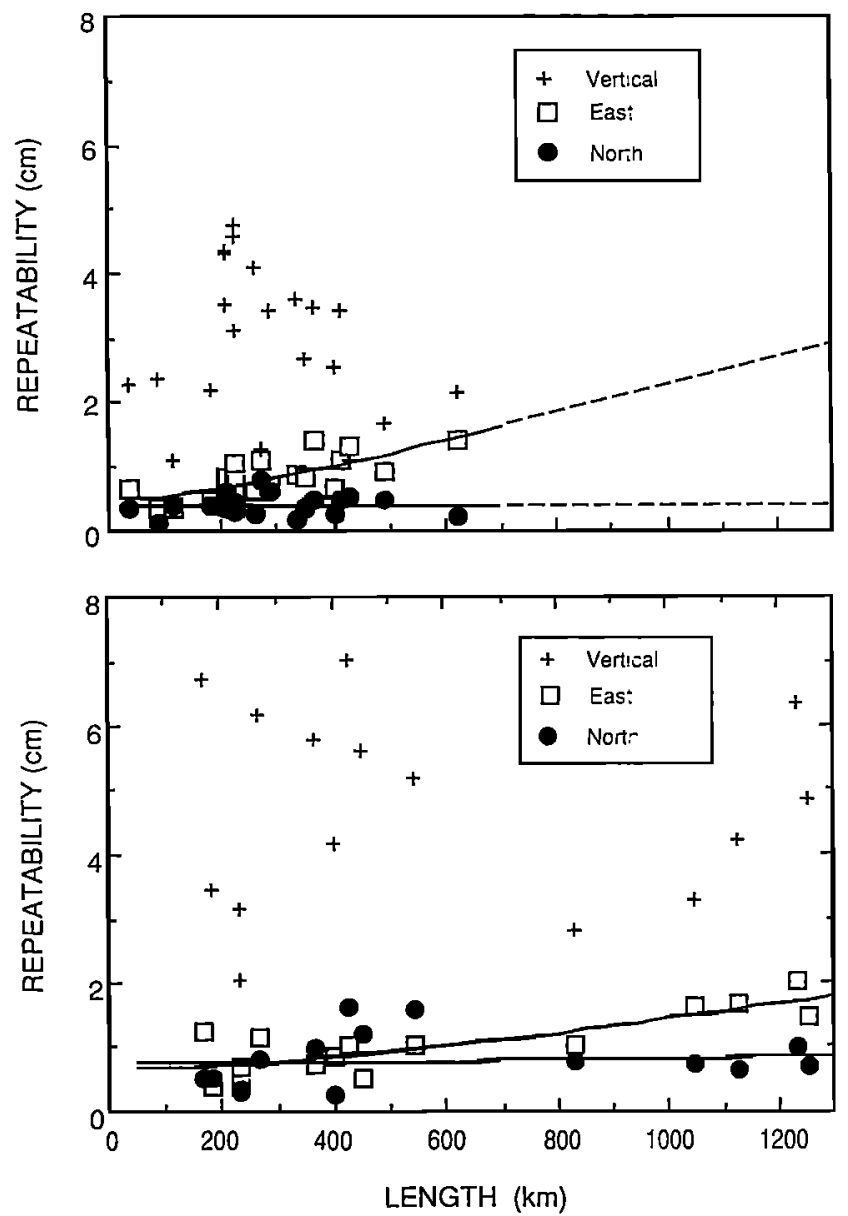

Figure 9. Short-term (3-8 days) repeatability (equation (30)) for experiments conducted in 1986 in southem Califomia (top) and the northern Caribbean (bottom). Curves are best fit (equation (31)) through horizontal components only. Note the higher scatter in the vertical component estimates for the northern Caribbean, in part reflecting higher levels of tropospheric calibration error [from Dixon et al., 1990, 1991a].

(on the Pacific plate), a baseline where VLBI data are also available. Straight-line fits through both data sets show northwest motion of Vandenberg with respect to Mojave, at a rate and direction broadly consistent with what we expect to see from a global plate model [DeMets et al., 1990]. The scatter of the GPS data about the best fit line is $6 \mathrm{~mm}$ (north), $10 \mathrm{~mm}$ (east), and $34 \mathrm{~mm}$ (vertical), not greatly different from the short-term repeatability noted in Figure 9 for a baseline of this length and in fact similar to the VLBI scatter [Dixon et al., 1990]. This suggests that at least in this location, for this period, the contribution of slowly varying systematic errors was low. Davis et al. [1989] investigated long-term $(-2$ year) repeatability for six short $(<50 \mathrm{~km})$ baselines and a single longer $(223 \mathrm{~km})$ baseline and found rms scatters about best fit lines of 2-12 mm (north), 2-19 $\mathrm{mm}$ (east), and 4-49 $\mathrm{mm}$ (vertical), where the larger values were for nonbias-fixed cases. Larson [1990b] presented data on long-term repeatability for 10 baselines between 91 and $464 \mathrm{~km}$ in length and found ms scatters over a 2-year period of 3-8 $\mathrm{mm}$ (north), 3-6 $\mathrm{mm}$ (east), and 11-33 mm (vertical). Thus it appears that for carefully configured experiments (for example, robust fiducial networks, observing sessions of adequate length, resolved carrier phase cycle ambiguities), the repeatability observed over several years is not significantly different from that observed over several days. These are encouraging results, suggesting that the magnitude of slowly varying systematic errors is similar to or less than the level of random errors manifested over several days.

The accuracy of any long baseline measurement is difficult to determine rigorously, since completely

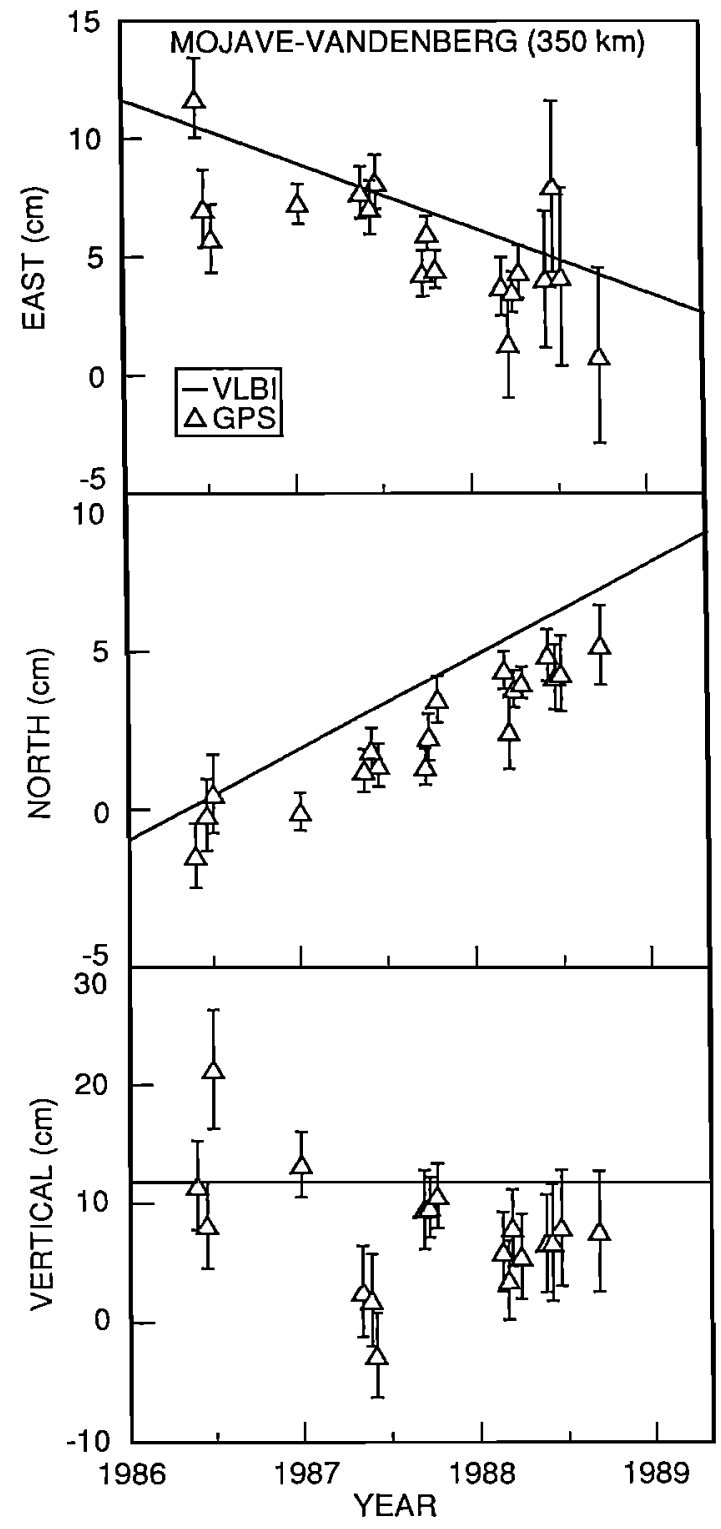

Figure 10. Comparison of GPS and VLBI results for the Mohave-Vandenberg baseline in Califomia between 1986 and 1988. Solid line is VLBI global solution GLB223; data from individual VLBI experiments omitted for clarity and have scatter similar to GPS data (from Larson [1990a] reprinted in the work by Dixon et al. [1990]). 
independent techniques of known accuracy are not available for comparison. However, if two independent techniques provide similar results, we can be more confident in each. For baselines $<50 \mathrm{~km}$ in length, conventional terrestrial geodetic techniques can be used. Davis et al. [1989] report comparisons with creep meters and alignment arrays on lines $\sim 10 \mathrm{~km}$ in length, suggesting GPS rate accuracies of $1-2 \mathrm{~mm} / \mathrm{yr}$. The same study described GPS comparisons with Geodolite trilateration data on lines in the range 10-50 km and found no detectable differences within the standard deviations of the measurements. For longer station separations, VLBI provides important data for comparison. However, VLBI and GPS are not completely independent, sharing, for example, a similar sensitivity to tropospheric effects. Also, GPS relies on Earth orientation data provided by VLBI, as well as fiducial site location data. Nevertheless, VLBIGPS comparisons allow the best available standard for GPS accuracy assessment for baselines longer than about $50 \mathrm{~km}$, and I will use the term accuracy to describe the results of GPS-VLBI comparisons, recognizing these limitations. Figure 11 [Dixon et al., 1990] shows differences between VLBI and GPS baseline estimates plotted as a function of baseline length for one experiment, with best fit curves (equation (31) and Figure 9) summarizing short-term repeatability in the same experiment. The comparison suggests that accuracy is very similar to the short-term repeatability, again limiting the magnitude of systematic error. Figure 10 suggests that it is possible to achieve a similar level of performance over longer time spans. Other GPS-VLBI comparisons in California have been reported: the 223-km Palos Verdes-Vandenberg baseline (six experiments between 1986 and 1988) [Davis et al., 1989]; the 245-km Owens Valley-Mojave baseline (a single experiment in 1987) [Dong and Bock, 1989], and the 224-km Palos Verdes-Mojave baseline (six experiments between 1986 and 1988) [Larson, 1990b]. All these comparisons indicate no significant source of systematic error of a type that would cause GPS-determined rates to differ from VLBI-determined rates. It is interesting to note, however, that comparisons involving GPS and fixed-site VLBI often exhibit near-constant offsets which fortunately do not affect the rate estimates. Figure 10 is a good example (see also Larson [1990b, Figure 4]. The best explanation for these offsets is that the site ties relating the phase centers of the large, fixed VLBI antennas to the nearby ground marks used by GPS may be in error. However, this hypothesis remains to be verified. Future work on GPS accuracy should also include GPS-SLR comparisons, since VLBI and GPS have some common error sources.

One problem with the two-parameter error model described by equation (31) is that dual-frequency GPS measurements have at least three significant error sources, each with a different dependence on baseline length. Receiver noise and set up errors are typically at the level of 1-2 mm, determined by "zero length" baseline tests, and are independent of baseline length. Atmospheric (mainly tropospheric) errors depend on baseline length only up to a certain distance, after which atmospheric variation is uncorrelated between two sites and the corresponding error reaches an approximately constant value, depending on calibration or estimation accuracy. The correlation length for troposphere-related errors at the level of a few millimeters, i.e., at a level exceeding receiver noise, will vary depending on site, season, and local weather but is likely to be on the order of tropospheric thickness, perhaps ranging from a few kilometers to a few tens of kilometers. For example, Ware et al. [1985] noticed significant differences in the wet delay for stations separated by $22 \mathrm{~km}$. Orbitrelated errors are expected to exhibit an almost linear dependence on baseline length up to several thousand kilometers, at a level of 1-2 parts in $10^{8}$ of baseline length

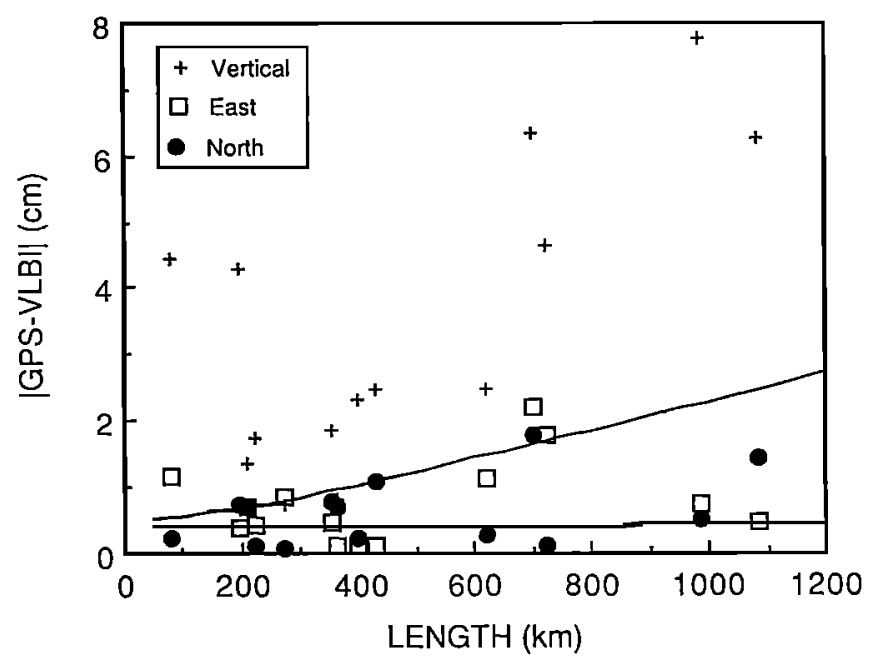

Figure 11. GPS results from an experiment in California in 1986, plotted as differences from VLBI solutions. Curves are best fit through short-term repeatability data from the same experiment (Figure 9) and are not based on the GPS-VLBI data. Note similarity between "precision" (based on repeatability curves) and "accuracy" (based on VLBI comparison) [from Dixon et al., 1990].

or better assuming good fiducial control. Note that for the range of baseline lengths typical of most GPS applications the projection of local vertical onto the horizontal baseline components due to Earth curvature is not critical to the error analysis. With these constraints in mind we can modify (31) to give a simple error model more applicable to GPS:

$$
\sigma=\left[a^{2}+b^{2}\left(1-e^{-L / \lambda}\right)^{2}+c^{2} L^{2}\right]^{1 / 2}
$$

where $a$ is the receiver and set up error, $b$ is the asymptotic tropospheric contribution to baseline error for station separations exceeding several tropospheric correlation lengths, $\lambda$ is the tropospheric correlation length, and $c$ is orbit-related error. An example model is shown in Figure 
12a. Note the crossover point at about $400 \mathrm{~km}$, where orbit errors start to dominate. Tropospheric error dominates for station separations longer than about $10 \mathrm{~km}$ and shorter than $400 \mathrm{~km}$ in this example. Figure 12 also shows some long-term repeatability data for a series of GPS experiments in California for comparison to the model. The caption lists the criteria used to minimize interexperiment differences, hopefully ensuring that data with similar error characteristics have been compared. However, by using long-term repeatability, we have undoubtedly averaged over a range of tropospheric conditions.

Most of the GPS-VLBI comparisons performed to date are restricted to a fairly limited region in the southwestern U.S., and a note of caution is in order when applying the resulting arguments on GPS precision and accuracy to other regions. Sensitivity studies indicate that fiducial network uncertainties are the largest contributor to systematic orbit and baseline error, the exact amount depending on experiment location and baseline length and orientation. Geodetic networks in the southwestern U.S. are less susceptible to this effect because of the proximal location of several high-quality fiducial sites, i.e., sites with a long history of VLBI occupations and wellestablished local ties between the VLBI antenna phase center and the GPS mark. Even so, uncertainties in VLBI-GPS ties at the $1-2 \mathrm{~cm}$ level are common [Tralli and Lichten, 1990; Dixon et al., 1991c]. The effect of these errors grows with distance from the fiducial network but will not cause increased scatter over several days, as the orbit geometry is essentially constant over this period. The effects may even be small over 1-2 year periods if the satellite constellation happens to be similar for several consecutive experiments, and if the same or similar fiducial networks are used. However, over the 5-10 year or longer periods likely to characterize many GPS experiments (see below), such fidelity is unlikely, implying the need for care in the establishment and maintenance of fiducial networks.

When we perform a series of GPS experiments, we generally wish to obtain estimates of strain rate or fault motion rate. Many geologic problems require rate accuracies in the range $1-5 \mathrm{~mm} / \mathrm{yr}$ [Jordan and Minster, 1988]. In order to plan experiments it is important to know how long it will take to acquire a data set with a given rate accuracy, or how often an experiment must be repeated within a fixed period (for example, 5-10 years) to achieve that accuracy. The uncertainty in a rate estimate $\sigma_{r}$ is a function of the single measurement position accuracy $\sigma_{m}$, the interval between experiments $\Delta t$ (assumed constant in the relation below), and the total time span of observations, $T$, and can be estimated from [Coates et al., 1985]:

$$
\sigma_{r}=\frac{\sigma_{m}}{T}\left[\frac{12 T / \Delta t}{(1+T / \Delta t)(2+T / \Delta t)}\right]^{1 / 2}
$$
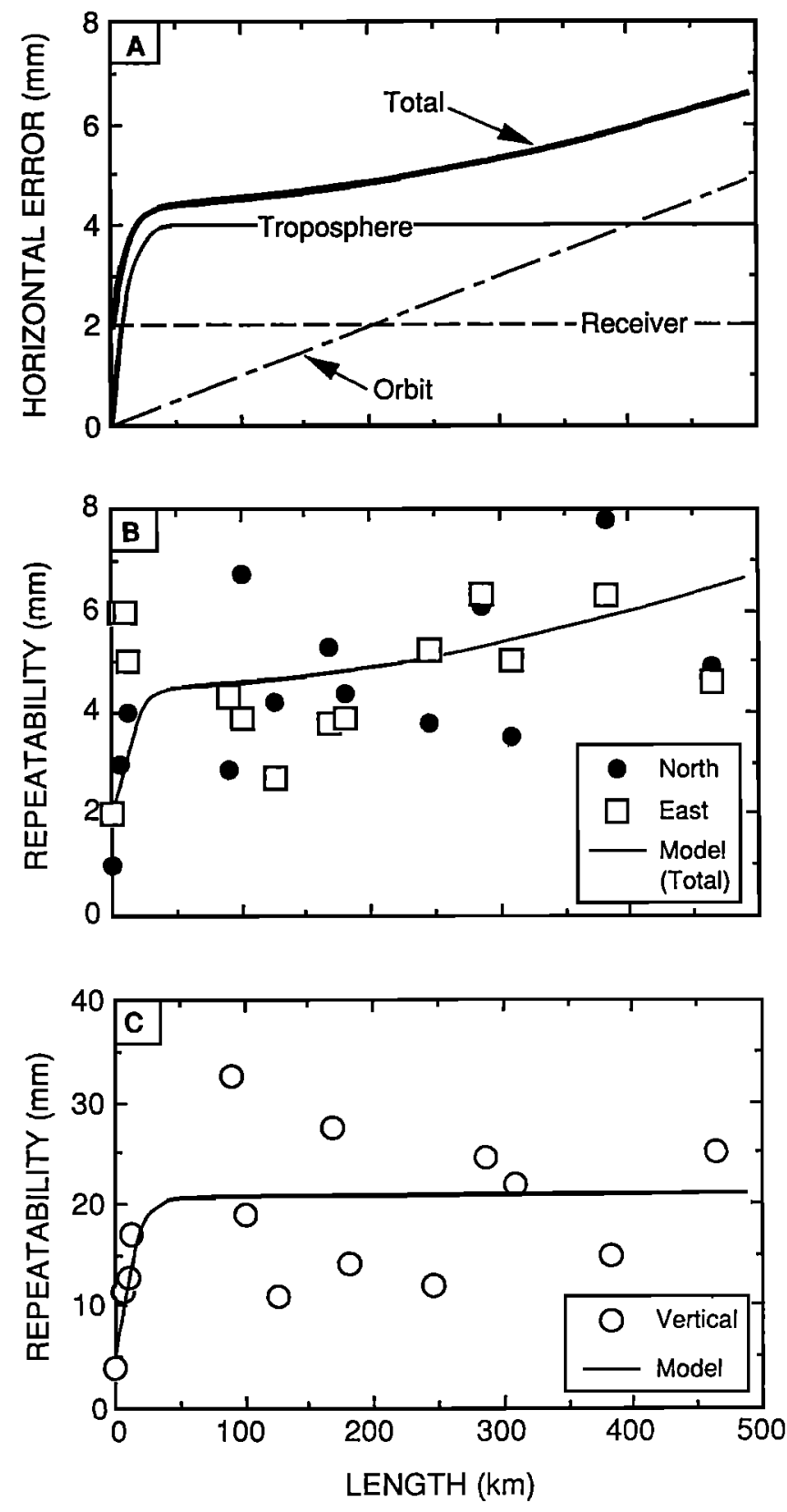

Figure 12. (a) Individual error components and total (rss) error from equation (32) for the horizontal component of a hypothetical GPS experiment, with $a=2 \mathrm{~mm}$ ("receiver"), $b=4 \mathrm{~mm}, \lambda=$ $10 \mathrm{~km}$ ( $b$ and $\lambda$ together define "troposphere"), and $c=1 \times 10^{-8}$ ("orbit"). (b) Long-term repeatability (weighted rms scatter about a best fit straight line) for GPS horizontal component measurements in central and southern Califomia between 1986 and 1988 compared to an error model (equation (32)). All data plotted are from TI-4100 receivers, represent four or more experiments, and have all or most carrier phase biases resolved. Data are from Davis et al. [1989] and Larson [1990b]. "Model" curve is same as "total" in Figure 12a. (c) Similar to Figure 12b, for vertical component. Model curve from equation (32)), with $a=5 \mathrm{~mm}, b$ $=20 \mathrm{~mm}, \lambda=10 \mathrm{~km}$, and $c=1 \times 10^{-8}$. 
Figure 13 plots the rate uncertainty $(1 \sigma)$ for single measurement uncertainties of 1 and $2 \mathrm{~cm}(1 \sigma)$, for several values of $\Delta t$. While the single measurement uncertainty is clearly important, the most efficient way to reduce the uncertainty in a rate estimate is to extend the total time span of observations. Comparison of modem GPS or other geodetic data and lower quality historical geodetic data taken $\mathbf{2 0}$ or more years ago is a good example of this principle. Significant compressional deformation along parts of the southern San Andreas fault system in California has been documented with this approach [Feigl et al., $1990 b ; W e b b, 1990]$.

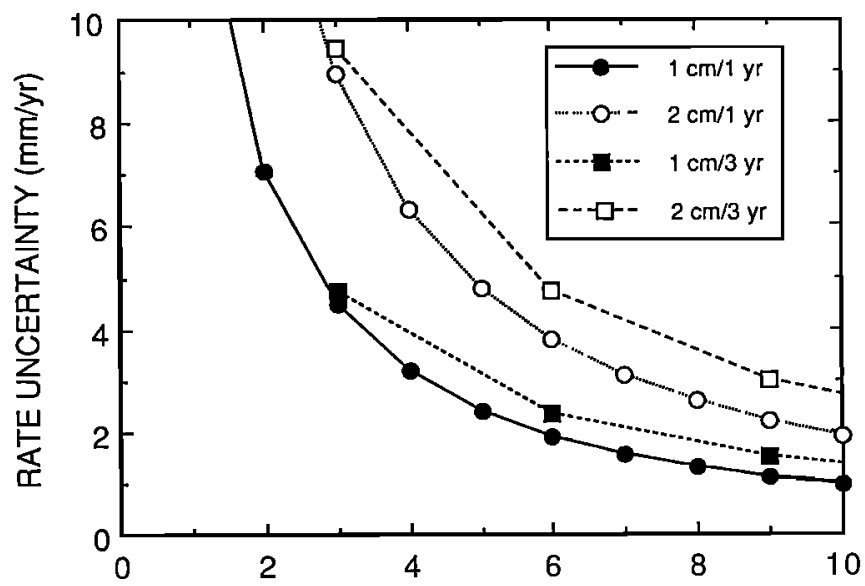

TIME AFTER FIRST EXPERIMENT (yrs)

Figure 13. Uncertainty of a geodetically determined rate estimate as a function of time, $T$ (equation (33)) for two values of single measurement uncertainty, $\sigma_{m}$ ( 1 and $2 \mathrm{~cm}$, listed first in legend) and two values of experiment repeat interval, $\Delta t(1$ and 3 years, listed second). Note that rate uncertainty is better than $3 \mathrm{~mm} / \mathrm{yr}$ after 10 years for all cases shown.

\section{GEOLOGICAL APPLICATIONS AND ACCURACY REQUIREMENTS}

In this section we review several applications of GPS and their corresponding accuracy requirements. The examples encompass a wide range of station separations, an important factor in determining both the accuracy achievable with GPS and critical aspects of experiment design.

Figure 14 (modified from Minster et al. [1990] shows the temporal and spatial scales of a variety of geological phenomena where geodetic monitoring can provide useful information. The regions of applicability for several geodetic techniques are also shown. It is apparent that even at current levels of performance, GPS can contribute to the study of a wide variety of phenomena. This figure also indicates a rough estimate of GPS performance 5 years hence, based on the expected impact of some recent developments.
In the limit of short length and time scales (implying dense networks and frequent observations), high-accuracy GPS is currently limited mainly by economic and logistical constraints, receiver limitations, and data processing efficiency. In the near future, with receiver improvements and the enhanced constellation, $\sim 5-\mathrm{mm}$ horizontal accuracy should be achievable over a wide range of station separations with kinematic or rapid static techniques, enabling dense spatial sampling. As receiver costs fall and the efficiency of data analysis improves, continuous monitoring of dense networks with GPS also becomes feasible, allowing greatly improved temporal sampling. This capability will complement seismic networks and strain observatories (D. M. Tralli, Spectral comparison of continuous Global Positioning System and strainmeter measurements of crustal deformation, submitted to Geophysical Research Letters, 1991), enabling monitoring of phenomena at periods just beyond the limit of longperiod seismometers, for example, creep and strain events (Figure 14) and other possible effects related to stress migration. Since $1-\mathrm{mm}$ horizontal precision in theory is achievable with several minute sampling of carrier phase and precise $P$ code pseudorange data over short $(<10 \mathrm{~km})$

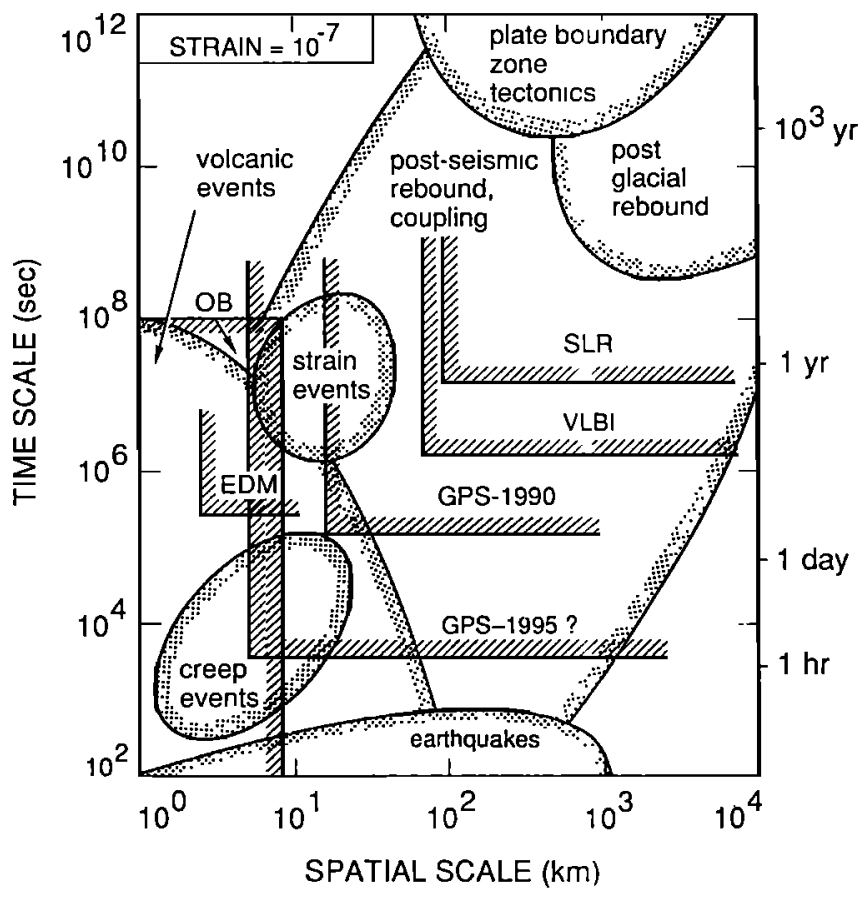

Figure 14. Cartoon showing order of magnitude estimates for spatial and temporal scales of some geological phenomena (lower case letters, stipple pattern) compared to detection capability of some geodetic techniques (upper case letters, diagonal line pattem), assuming $10^{-7}$ strain or higher and typical operating conditions. $O B$, observatory instruments (for example, continuously recording strainmeters, tiltmeters); VLBI, very long baseline interferometry; SLR, satellite laser ranging; EDM, two color laser electronic distance measuring equipment, assuming daily measurements. Modified from Minster et al., [1990]. 
baselines, it is tempting to speculate on the detectability of seismic precursors proposed by Lorenzetti and Tullis [1989] with a dense array of continuously operating GPS stations.

In the limit of long length scales, GPS accuracy will improve as global tracking networks evolve to include a uniform set of well-distributed, high-accuracy ground systems with strict configuration control, and as force models for solar radiation pressure and other orbit perturbations are refined. This will enable a number of plate motion and regional plate boundary zone problems to be attacked. Even with current performance there are many more applications of GPS than we can address here, but to give some flavor for the diversity, I will consider two types of applications, divided arbitrarily by length scale.

\section{Short (0-100 km) Baselines: The Near Field}

Geodetic measurements within about $50-100 \mathrm{~km}$ of a fault system may provide constraints on crust and mantle rheology, faulting mechanics, and the earthquake cycle. This length scale is also appropriate for monitoring upper crustal deformation associated with inflation/deflation of shallow magma chambers. Until recently, most such studies were performed by conventional terrestrial geodetic techniques (triangulation, trilateration distance measurement, and leveling). The accuracy, site flexibility, ease of data acquisition, and increasingly straightforward data analysis allows GPS to complement, and in some cases, replace these approaches. The accurate three-dimensional vector nature of GPS positioning also allows constraints to be placed on block rotations, something that is not possible with conventional techniques alone [e.g., Davis et al., 1989]. Minster et al. [1990], Bilham [1991], and Hager et al. [1991] review application of geodetic strain determination to near-field and earthquake studies. In this section I will focus mainly on one aspect, namely the accuracy required for GPS data to distinguish among some plausible models of faulting and crustal rheology.

A series of GPS experiments allows determination of surface strain and strain rate tensors, reflecting stresses and theological properties in the crust and mantle. Simple elastic half-space models and more complicated rheological distributions have been used to explain observed surface deformation [e.g., Melosh, 1976, 1977; Savage and Prescott, 1978; Spence and Turcotte, 1979; Turcotte et al., 1984; $\mathrm{Li}$ and Rice, 1987; Li and Lim, 1988]. The manner in which deformation changes in space (away from a fault) and time (before and after a large earthquake) may reveal information about the rheology of the lower layers but may also require large numbers of accurate measurements on any given fault segment to describe adequately the spatial and temporal distribution of strain. Important questions include whether GPS data allow discrimination among these models, what accuracy is required, and whether we need to incorporate any extraordinary features into the GPS experiment design?

Consider the surface strain pattern for a locked, vertical strike-slip fault (strain models appropriate to convergence zone thrust faults are given by Savage [1983] and Thatcher and Rundle [1984]). The fault-parallel surface displacement rate $v$ during an earthquake cycle for a simple elastic half-space model is [Savage and Burford, 1973]:

$$
\nu=\left(v_{0} / \pi\right) \tan ^{-1}(x / D)
$$

where $D$ is the fault or "locking" depth related to the brittle-ductile transition depth, $x$ is distance perpendicular to the fault, and $v_{0}$ is the total slip rate at large distances from the fault or beneath the locking depth. If $T$ is the earthquake recurrence interval, then the coseismic slip that relieves accumulated elastic strain (and stress) is just $v_{0} T$. Geodetic data may be inverted for $D$ and $v$. Constraints on $D$ are also available from seismicity (large earthquakes tend to nucleate near the base of the fault, at the brittleductile transition), and $v_{0}$ may be determined from far-field space geodetic measurements (VLBI, SLR, or GPS) or, for a simple plate boundary, from a global plate motion model. For strike-slip faults in continental crust, $D$ is typically about $10-20 \mathrm{~km}$, on the basis of inversion of geodetic data [e.g., King et al., 1987], seismic depth data, and rheological properties of crustal materials from laboratory experiments [Sibson, 1983]. Figure 15 plots differences in fault-parallel displacement rates for elastic half-space models with $D=10,15$, and $20 \mathrm{~km}$. The largest rate differences are observed at horizontal distances from the fault roughly equal to fault depth, giving some guidance as to station placement. Distinguishing among the models would require resolution of fault-parallel displacement rates at the $1-3 \mathrm{~mm} / \mathrm{yr}$ level.

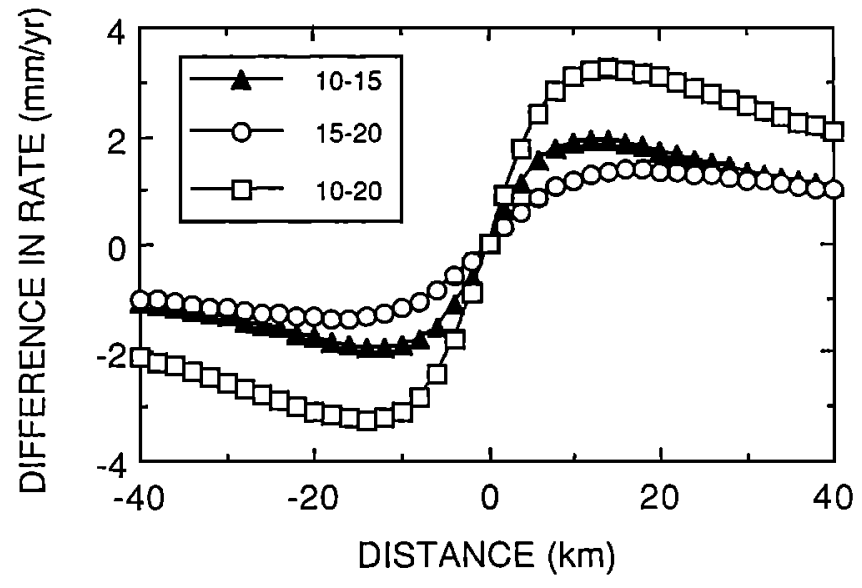

Figure 15. Difference in fault parallel surface displacement rate for several models assuming a locked, vertical strike-slip fault in an elastic half space (equation (34)) for fault depths, $D=10,15$, and $20 \mathrm{~km}$. A far field displacement rate of $30 \mathrm{~mm} / \mathrm{yr}$ is assumed.

A more realistic model for deformation near a fault accounts for the layered rheology of the crust and upper mantle and the coupling that occurs between layers. Recent models assume that lower layer or layers behave like Maxwell viscoelastic solids. Like its mechanical analogue, 
a spring and dashpot in series, a viscoelastic substance behaves elastically over short time scales and viscously over long time scales. Viscous relaxation in the viscoelastic "asthenosphere" damps short-term perturbations in the overlying elastic "lithosphere." The rate of stress relaxation is related to a time constant $2 \eta / \mu$, where $\eta$ is the viscosity of the lower layer and $\mu$ is the average modulus of rigidity of the two layers. Expressions for the surface strains predicted by coupling models are given by Savage and Prescott [1978], Turcotte et al. [1984], and $L i$ and Rice [1987]. Inspection of these models suggests that differences in fault-parallel surface displacement rates of 1-2 $\mathrm{mm} / \mathrm{yr}$ or better are required for discrimination. However, for most parts of the earthquake cycle, predicted surface deformation with the elastic half-space model and even the simplest (two layer) viscoelastic coupling model may be surprisingly similar and thus difficult to discriminate with GPS or any other geodetic technique alone [Savage and Prescott, 1978; Thatcher, 1983; Savage, 1990]. On the other hand, time-varying signals over part of the earthquake cycle are a potentially useful observable [Thatcher, 1983; Li and Rice, 1987], and we should not rely on long ( $>10-15$ years) observation times to achieve a given rate accuracy. Davis et al. [1989] obtained rate uncertainties of $1 \mathrm{~mm} / \mathrm{yr}$ in horizontal components and 3-4 $\mathrm{mm} / \mathrm{yr}$ in the vertical component for baselines up to $12 \mathrm{~km}$ in length in 3 years using GPS data taken several times per year.

GPS is also well suited to define the coseismic strain field associated with earthquakes. These strains may exhibit great spatial variation within a few tens of kilometers of the fault, particularly for complex fault geometry, implying the need for dense surveys. It may be useful to separate coseismic rupture from postseismic relaxation phenomena, implying a requirement for rapid response and frequent resurvey after an event. If required accuracies are not high (several centimeters are sufficient for most studies of coseismic offset), kinematic or rapid static techniques enabling survey of large numbers of points may be useful. Larsen [1990] used kinematic techniques to measure coseismic offsets for the 1987 Superstition Hills earthquake sequence in southern California.

\section{Long Baselines $(100 \geq 1000 \mathrm{~km})$}

Long baseline measurements with GPS are challenging because the influence of orbit errors is directly proportional to baseline length, and because carrier phase cycle ambiguity resolution becomes more difficult at station separations exceeding $100-200 \mathrm{~km}$. Special care is required in network design, and covariance analyses prior to the actual experiment may be important. The geologic rationale for pursuing such difficult GPS experiments, some examples, and preliminary results are given below.

Relative velocities of most of the major plates are now well known from global geologic models, with precisions of a few millimeters per year or better in rate and a few degrees or better in azimuth [DeMets et al., 1990]. These models are based on spreading rates from mid-ocean ridges, determined from magnetic anomalies averaged over several million years, azimuths of transform faults that average over a similar time interval, and earthquake slip vectors representing essentially instantaneous local azimuth information. There is no reason to suspect that plate motion averaged over several million years differs over shorter (for example, geodetic) time scales when measured between plate interiors, away from the transient effects of plate boundary deformation, unless a plate boundary is undergoing rapid evolution. This hypothesis has been largely confirmed by NASA's Crustal Dynamics Project, which has made velocity determinations between several major plates with VLBI [e.g., Argus and Gordon, 1990] and SLR [e.g., Harrison and Douglas, 1990]. Nevertheless, there are some plate boundaries where relative plate motion is poorly constrained, and where GPS (as well as VLBI and SLR) measurements can make unique contributions. Plates lacking a spreading center boundary (for example, the Philippine plate) or with only small or poorly defined spreading center boundaries (for example, the Caribbean plate) will have poorly defined relative motion rates with respect to adjacent plates. Plate boundaries where transform fault trends or earthquake slip azimuths may be biased due to the influence of heterogeneous continental crust (for example, the PacificNorth America plate boundary north of the southern Gulf of California) will have poorly determined relative motion azimuths. Predicted motion at subduction zones may be biased because of systematic biases in trench earthquake slip vectors [DeMets et al., 1990]. In cases where local data are lacking or biased, relative plate motion is determined mainly from closure conditions on other plates, and GPS or other space geodetic technique can provide useful complementary information. GPS stands out as the appropriate technique if economic or logistical considerations preclude the use of SLR or VLBI, both of which have larger, more cumbersome ground systems, and if the problem can be solved with station separations less than $1000-2000 \mathrm{~km}$ (both VLBI and SLR have better performance at longer station separations).

Figure 16 shows the uncertainty in relative velocities of some plate pairs with convergent boundaries. If at least one of the plates is oceanic, nearby measurement sites spanning the trench plate boundary may not exist. One of the goals of the CASA (Central and South America) GPS experiments [Kellogg et al., 1989; Kellogg and Dixon, 1990] is to determine the rate and direction of subduction of the Cocos and Nazca plates beneath Central and northern South America, among the most poorly determined plate pairs in the NUVEL 1 plate motion model [DeMets et al., 1990] (Figure 16). This requires measurements from Cocos Island (the only point of land on the Cocos plate) and Malpelo Island (the only point of land on the Nazca plate within $500 \mathrm{~km}$ of mainland South America) to points in Central and South America, defining numerous long 


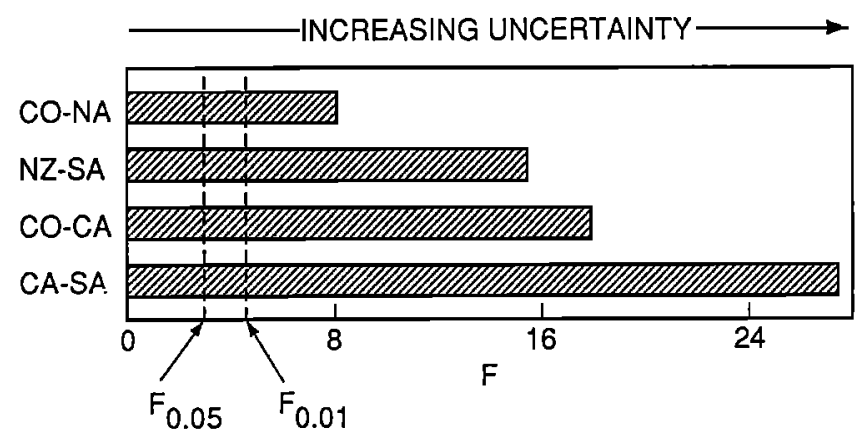

Figure 16. Uncertainty in relative plate motion vectors (NUVEL 1 model) [DeMets et al., 1990] for plate pairs where local motion is constrained mainly by trench slip vectors; most other plate pairs in the model are better determined. Uncertainty is based on the $F$ ratio test for plate closure [Gordon et al., 1987]. " $F .01$ " and " $F .05$ " indicate $1 \%$ and $5 \%$ risk levels, respectively $(99 \%$ and 95\% confidence levels). CO, Cocos; NA, North America; NZ, Nazca; SA, South America; CA, Caribbean. Modified from DeMets et al. [1990].

baselines. It was known in advance that a robust fiducial network would be required to perform such measurements, and covariance analyses were performed to determine optimal configurations [Freymueller and Golombek, 1988]. These analyses suggested that a North American fiducial network by itself was inadequate. A global network of tracking stations was instituted for this experiment, the first civilian global GPS network [Neilan et al., 1989]. Preliminary results using this network tend to confirm the predictions of the covariance analyses, with significant improvements in long baseline estimates when the expanded tracking network is used [Kornreich Wolf et al., 1990; Freymueller and Kellogg, 1990; Figure 5). The second CASA experiment is scheduled for 1991.

Relative motion between plates is occasionally accommodated within a continent. Continental crust is both weaker and older on average than oceanic crust, and inherited weaknesses of varying orientation may be reactivated under the newer stress regime of the plate boundary [Ivins et al., 1990], complicating the boundary's geometry and evolution, and often leading to broad zones of distributed deformation. Examples of complex continental transform boundaries include the San Andreas fault zone in California, the Motogua-Polochic fault zone in Central America, and the Alpine fault zone in New Zealand. High-precision GPS measurements can be useful in characterizing both the integrated motion across the entire deformation zone and details of crustal behavior near individual faults within the plate boundary zone, including rotations of quasi-rigid blocks between faults. Knowledge of individual fault rates and the total plate rate can lead to development of internally consistent kinematic block models. In effect, a complete mass balance can be obtained because adequate spatial sampling is possible with GPS. For example, the southern San Andreas system has at least three major faults that are important in accommodating the tangential components of relative plate motion between the Pacific and North American plates. Rotations are predicted theoretically [Ivins, 1989] and Neogene rotations in parts of the plate boundary zone have been observed with paleomagnetic and other data [Carter et al., 1987; Luyendyk and Hornafius, 1987; Nur et al., 1989]. Kinematic models of the region [e.g., Bird and Rosenstock, 1984; Weldon and Humprheys, 1986; Rundle, 1986] are being tested with GPS, employing a network that includes islands of the southern Califomia borderland and station separations mainly in the range $100-500 \mathrm{~km}$ [Agnew et al., 1988; Dixon et al., 1990]. Precision of the GPS-based rate estimates should be commensurate with the models (a few millimeters per year) [Bird and Rosenstock, 1984; Jordan and Minster, 1988], achievable in 5 years with yearly GPS measurements (Figure 13). Preliminary results are reviewed by Hager et al. [1991].

Total relative motion between the Pacific and North America plates can be measured by spanning the relatively simple ridge-transform boundary in the southern Gulf of California, avoiding tectonic complexities associated with the Transverse Ranges and Basin and Range extension. The relevant baselines are $300-500 \mathrm{~km}$ in length. GPS measurements were made in 1985 and 1989, allowing a preliminary determination of the relative motion vector (Figure 17). Using a sensitivity analysis to quantify random and systematic error, Dixon et al. [1991c] showed that the Pacific-North America relative motion vector determined by GPS in the southern Gulf of California is consistent, within $1 \sigma$ total error $\left( \pm 7 \mathrm{~mm} / \mathrm{yr}\right.$ in rate, $\pm 6^{\circ}$ in azimuth), to the NUVEL 1 global model [DeMets et al., 1990].

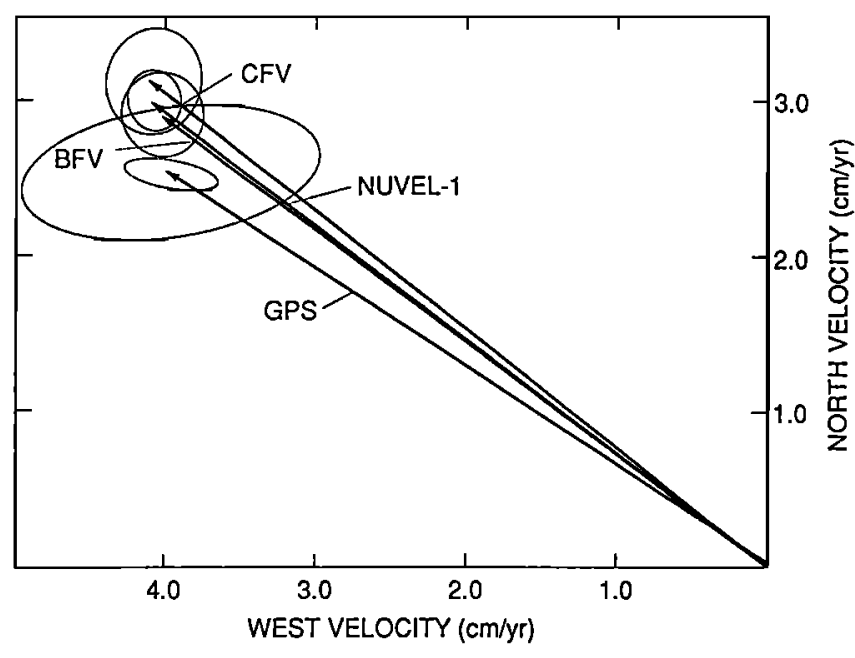

Figure 17. Vector diagram summarizing relative motion of Pacific plate (at Cabo San Lucas) with respect to North America, based on GPS measurement, and NUVEL 1 global vector, closure fitting vector (CFV), and best fitting vector (BFV) [DeMets et al., 1990]. Error ellipses are $1 \sigma$ from Dixon et al. [1991]. 


\section{CONCLUSIONS}

I have covered in a general way the major principles behind GPS, particularly those relevant to high-precision geological and geophysical applications. GPS is an impressive new measurement tool; the rate of improvement in the quality and quantity of geodetic measurements with GPS over the last decade is astonishing. We can expect to see continued improvements in receiver accuracy and portability, reduced receiver cost, decreased time and cost for data processing, improved models and calibration of major error sources, corresponding increases in the number of diversity of scientific applications, and some very interesting scientific results.

ACKNOWLEDGMENTS. Comments on a preliminary version of the manuscript by Eric Ivins, Steve Lichten, and Larry Young were very helpful. I am indebted to Jim Davis and Bob King for thorough reviews, and editor-in-chief Marcia Neugebauer for patience and many helpful suggestions. Ken Hurst, Chris Rocken, Dave Tralli, and Bob King kindly provided manuscripts in advance of publication. This work was conducted at the Jet Propulsion Laboratory, California Institute of Technology, under contract to NASA.

Marcia Neugebauer was the editor responsible for this paper. She thanks James Davis and Robert King for their assistance in evaluating the technical content and Brian Tinsley for serving as a cross-disciplinary referee.

\section{REFERENCES}

Abbot, R. I., and C. C. Counselman, Demonstration of GPS orbit determination enhancement by resolution of carrier phase ambiguity (abstract), Eos Trans. AGU, 68, 1238, 1987.

Agnew, D. C., Y. Bock, R. King, T. Jordan, B. Hager, and D. Jackson, GPS measurements in central and southern California, CSTG Bull., 10, 71-87, 1988.

Allan, D. W., Statistics of atomic frequency standards, Proc. IEEE , 54, 221-230, 1966.

Allan, D. W., M. A. Weiss, and T. K. Peppler, In search of the best clock, IEEE Trans. Instrum. Meas., 38, 624-630, 1989.

Aoki, S., B. Guinot, G. H. Kaplan, H. Kinoshita, D. D. McCarthy, and P. K. Seidelman, The new definition of universal time, Astron. Astrophys., 105, 359-361, 1982.

Argus, D. F., and R. G. Gordon, Comparison of Pacific-North America plate motion determined from very long baseline interferometry with that determined from magnetic anomalies, transform faults and earthquake slip vectors, J. Geophys. Res., 95(11), 17,315-17,324, 1990.

Beutler, G., W. Gurtner, I. Bauersima, and M. Rothacher, Efficient computation of the inverse of the covariance matrix of simultaneous GPS carrier phase difference observations, Man. Geod., 11, 249-255, 1986.

Beutler, G., I. Bauersima, W. Gurtner, M. Rothacher, and T. Schildknecht, Evaluation of the 1984 Alaska Global Positioning System campaign with the Bernese GPS software, $J$. Geophys. Res., 92, 1295-1303, 1987.

Bevis, M., GPS networks: The practical side, Eos Trans. AGU, 72, 49-56, 1991.
Bilham, R., Earthquakes and sea level: Space and terrestrial metrology on a changing planet, Rev. Geophys., 29(1), 1-29, 1991.

Bird, P. and R. W. Rosenstock, Kinematics of present crust and mantle flow in Southem Califomia, Geol. Soc. Am. Bull., 95, 946-957, 1984.

Black, H. D., and A. Eisner, Correcting satellite Doppler data for tropospheric effects, J. Geophys. Res., 89, 2616-2626, 1984.

Blewitt, G., Carrier-phase ambiguity resolution for the Global Positioning System applied to geodetic baselines up to 2000 km, J. Geophys. Res., 94, 10,187-10,203, 1989.

Blewith, G., GPS techniques for monitoring geodynamics at regional scales, paper presented at Second International Symposium on Precise Positioning with Global Positioning System, Int. Assoc. of Geod., Ottawa, 1990.

Blewitt, G., U. J. Lindqwister, and K. W. Hudnut, Densification of continuously operating GPS arrays using a rapid static survey technique (abstract), Eos Trans. AGU, 70, 1054, 1989.

Bock, Y., R. I. Abbot, C. C. Counselman III, R. W. King, and S. A. Gourevitch, Three-dimensional geodetic control by interferometry with GPS: Processing of GPS phase observables, paper presented at First International Symposium on Precise Positioning With Global Positioning System, Int. Assoc. of Geod., Rockville, Md., 1985a.

Bock, Y., R. I. Abbot, C. C. Counselman III, S. A. Gourevitch, and R. W. King, Establishment of three-dimensional geodetic control by interferometry with the Global Positioning System, J. Geophys. Res., 90, 7689-7703, $1985 b$.

Bock, Y., S. A. Gourevitch, C. C. Counselman III, R. W. King, and R. I. Abbot, Interferometric analysis of GPS phase observations, Man. Geod., 11, 282-288, 1986.

Bossler, J. D., C. C. Goad, and P. L. Bender, Using the Global Positioning System (GPS) for geodetic positioning, Bull. Geod., 54, 553-563, 1980.

Carter, J. N., B. P. Luyendyk, and R. Terres, Neogene clockwise tectonic rotation of the Eastem Transverse Ranges, California, Geol. Soc. Am. Bull., 98, 199-206, 1987.

Carter, W. E., D. S. Robertson, and J. R. Mackay, Geodetic radio interferometric surveying: Applications and results, $J$. Geophys. Res., 90, 4577-4587, 1985.

Chao, C. C., The troposphere calibration model for Mariner Mars 1971, JPL Tech. Rep., 32-1587, 61-76, 1974.

Clark, T. A., W. G. Melboume, C. Reigber, L. E. Young, and T. P. Yunck, Microwave Techniques, in The Interdisciplinary Role of Space Geodesy, vol. 22, edited by I. I. Mueller and S. Zerbini, p. 43-102, Springer-Verlag, New York, 1989.

Coates, R. J., H. Frey, G. D. Mead, and J. M. Bosworth, Space age geodesy: The NASA Crustal Dynamics Project, IEEE Trans. Geosci. Remote Sens., GE-23, 360-368, 1985.

Cohen, S. C., and D. E. Smith, LAGEOS scientific results: Introduction, J. Geophys. Res., 90, 9217-9220, 1985.

Counselman, C. C., and S. Gourevitch, Miniature interferometer terminals for Earth surveying: Ambiguity and multipath with the Global Positioning System, IEEE Trans. Geosci. Remote Sens., GE-19, 244-252, 1981.

Counselman, C. C., and I. I. Shapiro, Miniature interferometer terminals for earth surveying, Bull. Geod., 53, 139-163, 1979.

Davis, J. L., T. A. Herring, I. I. Shapiro, A. E. E. Rogers, and G. Elgered, Geodesy by radio interferometry: Effects of atmospheric modeling errors on estimates of baseline length, Radio Sci., 20, 1593-1607, 1985.

Davis, J. L., W. H. Prescolt, J. L. Svarc, and K. Wendt, Assessment of Global Positioning System measurements for studies of crustal deformation, J. Geophys. Res., 94, 13,635-13,650, 1989.

DeMets, C., R. G. Gordon, D. F. Argus, and S. Stein, Current plate motions, Geophys. J. Int., 101, 425-478, 1990.

Dickey, J. O., XX Newhall, and J. G. Williams, Earth orientation data from lunar laser ranging and an error analysis of polar motion services, J. Geophys Res., 90, 9353-9362, 1985. 
Dixon, T. H., and S. Komreich Wolf, Some tests of wet tropospheric calibration for the CASA Uno Global Positioning System experiment, Geophys. Res. Lett., 17, 203-206, 1990.

Dixon, T. H., M. P. Golombek, and C. L. Thomton, Constraints on Pacific plate kinematics and dynamics with Global Positioning System (GPS) measurements, IEEE Geosci. Remote Sens., GE-23, 491-501, 1985.

Dixon, T. H., G. Blewitt, K. Larson, D. Agnew, B. Hager, P. Kroger, L. Scrumeda, and W. Strange, Global Positioning System measurements of crustal deformation in southem Califormia: Some constraints on performance, Eos Trans. $A G U, 71,1051-1056,1990$.

Dixon, T. H., G. Gonzalez, S. Lichten, and E. Katsigris, First epoch geodetic measurements with the Global Positioning System across the northern Caribbean plate boundary zone, $J$. Geophys. Res., 96, 2397-2415, 1991 a.

Dixon, T. H., D. M. Tralli, G. Blewith and J. P. Dauphin, Geodetic baselines across the Gulf of California using the Global Positioning System, Am. Assoc. Petrol. Geol., in press, $1991 b$.

Dixon, T. H., G. Gonzalez, S. Lichten, D. Tralli, G. Ness, and J. P. Dauphin, Preliminary determination of Pacific-North America Plate motion in the southem Gulf of Califomia with the Global Positioning System, Geophys. Res. Lett., in press, $1991 c$.

Dong, D., and Y. Bock, GPS network analysis with phase ambiguity resolution applied to crustal deformation studies in California, J. Geophys. Res., 94, 3949-3966, 1989.

Easton, R. L., The Navigation Technology Program, Nav. J. Inst. Nav., 25, 107-120, 1978.

Elgered, G., Tropospheric wet path delay measurements, IEEE Trans. Antennas Propag., AP-30, 502-505, 1982.

Elgered, G., J. L. Davis, T. A. Herring, and I. I. Shapiro, Geodesy by radio interferometry: water vapor radiometry for estimation of the wet delay, J. Geophys. Res., in press, 1991.

Essen, L., and J. V. L. Parry, An atomic standard of frequency and time interval, Nature, 176, 280-282, 1956.

Feigl, K. L., R. W. King, T. A. Herring, and M. Rothacher, A scheme for reducing the effect of selective availability on precise GPS carrier phase measurements (abstract), Eos Trans. $A G U, 71,1268,1990 a$.

Feigl, K. L., R. W. King, and T. H. Jordan, Geodetic measurement of tectonic deformation in the Santa Maria fold and thrust belt, California, J. Geophys. Res., 95, 2679-2699, $1990 b$.

Fleigel, H. F., A. A. Feess, W. C. Layton, and N. W. Rhodus, The GPS radiation force model, paper presented at First International Symposium on Precise Positioning With Global Positioning System, Int. Assoc. of Geod., Rockville, Md., 1985.

Freymueller, J., and M. Golombek, Geometry and treatment of fiducial networks: Effect on GPS baseline precision in South America, Geophys. Res. Lett., 15, 1467-1469, 1988.

Freymueller, J., and J. N. Kellogg, The extended tracking network and indications of baseline precision and accuracy in the North Andes, Geophys. Res. Lett., 17, 207-210, 1990.

Goad, C. C., Precise relative position determination using Global Positioning System carrier phase measurements in a nondifference mode, paper presented at First International Symposium on Precise Positioning With Global Positioning System, Int. Assoc. of Geod., Rockville, Md., 1985.

Goad, C. C., and A. Mueller, An automated procedure for generating an optimum set of independent double difference observables using Global Positioning System carrier phase measurements, Man. Geod., 13, 365-369, 1988.

Gordon, R. G., S. Stein, C. DeMets, and D. F. Argus, Statistical tests for closure of plate motion circuits, Geophys. Res. Lett., $14,587-590,1987$.
Hager, B. H., R. W. King, and M. H. Murray, Measurement of crustal deformation using GPS, Ann. Rev. Geophys., in press, 1991.

Harrison, C. G. A., and N. B. Douglas, Satellite laser ranging and geologic constraints on plate motion, Tectonics, 9, 935-952, 1990.

Hellwig, H., Microwave time and frequency standards, Radio Sci., 14, 561-572, 1979.

Herring, T. A., Precision of vertical position estimates from very long baseline interferometry, J. Geophys. Res., 91, 9177-9182, 1986.

Herring, T. A., C. R. Gwinn, and I. I. Shapiro, Geodesy by radio interferometry: Studies of the forced nutations of the Earth, part 1, Data analysis, J. Geophys. Res., 91, 4745-4754, 1986.

Hide, R., and J. O. Dickey, The Earth's variable rotation, Science, in press, 1991.

Ivins, E. R., T. H. Dixon, and M. P. Golombek, Extensional reactivation of an abandoned thrust: $A$ bound on shallowing in the brittle regime, J. Struct. Geol., 12, 303-314, 1990.

Ivins, E. R., New aspects of rotational dynamics within the North-American-Pacific ductile shear zone, in Deep Structure and Past Kinematics of Accreted Terranes, Geophys. Monogr. Ser., vol. 50, edited by J. W. Hillhouse, pp. 179-201, AGU, Washington, D. C., 1989.

Janssen, M. A., A new instrument for the determination of radio path delay due to atmospheric water vapor, IEEE Trans. Geosci. Remote Sens., GE23, 485-490, 1985.

Jordan, T. H., and J. B. Minster, Beyond plate tectonics: Looking at plate deformation with space geodesy, in The Impact of VLBI on Astrophysics and Geophysics, edited by M. J. Reid and J. M. Moran, pp. 341-350, 1988.

Kaplan, G. H., The IAU resolutions of astronomical constants, time scales and the fundamental reference frame, Circ. 163 , US. Nav. Observ., Washington, D. C., 1981.

Kaula, W. M., Theory of Satellite Geodesy, 124 pp., Blaisdell, Waltham, Mass., 1966.

Kellogg, J., and T. H. Dixon, Central and South America GPS geodesy-CASA Uno, Geophys. Res. Lett., 17, 195-198, 1990.

Kellogg, J., T. H. Dixon, and R. Neilan, CASA: Central and South America GPS Geodesy-CASA, Eos Trans. AGU, 70, 649-656, 1989.

Kellogg, J. N., et al., First GPS baseline results from the North Andes, Geophys. Res. Lett., 17, 211-215, 1990.

King, N. E., P. Segall, and W. Prescott, Geodetic measurements near Parkfield, Califomia, 1959-1984, J. Geophys. Res., 92, 2747-2766, 1987.

King, R. W., and G. Blewitt, Present capabilities of GPS for high precision regional surveys, in Global Positioning System: An Overview, Int. Assoc. Geod. Symp. 102, edited by Y. Bock and N. Leppard, pp. 24-39, Springer-Verlag, New York, 1990.

King, R. W., E. G. Masters, C. Rizos, A. Stolz, and J. Collins, Surveying With Global Positioning System GPS, 128 pp., Dummler, Bonn, 1985.

Komreich Wolf, S., T. H. Dixon, and J. Freymueller, The effect of tracking network configuration on GPS baseline estimates for the CASA Uno experiment, Geophys. Res. Lett., 17, 647-650, 1990.

Lambeck, K., The Earth's Variable Rotation: Geophysical Causes and Consequences, Cambridge University Press, New York, 1980.

Lambeck, K., Geophysical Geodesy: The Slow Deformations of the Earth, 718 pp., Clarendon, Oxford, 1988.

Lanyi, G. E., Tropospheric delay effects in radio interferometry, JPL Prog. Rep., 42-78, 152-159, 1984.

Larson, K., Precision, accuracy and tectonics from the Global Positioning System, Ph.D. thesis, 269 pp., Univ. of Calif., San Diego, 1990a. 
Larson, K., Evaluation of GPS estimates of relative positions from central California, 1986-1988, Geophys. Res. Lett., 17, 2433-2436, $1990 b$.

Larsen, S., Global Positioning System measurements of deformation in southern Califomia, Ph.D. thesis, Calif. Inst. of Technol., 1990.

Lawson, C. L., and R. J. Hanson, Solving Least Squares Problems, 340 pp., Prentice-Hall, Englewood Cliffs, N. J., 1974.

Leick, A., GPS Satellite Surveying, 352 pp., Wiley-Interscience, New York, 1990.

Li, V. C., and J. R. Rice, Crustal deformation in great Califomia earthquake cycles, J. Geophys. Res., 92, 11,533-11,551, 1987.

Li, V. C., and H. S. Lim, Modelling surface deformation at complex strike-slip boundaries, J. Geophys. Res., 93, 7943-7954, 1988.

Lichten, S. M., High accuracy Global Positioning System orbit determination: Progress and prospects, in Global Positioning System: An Overview, Int. Assoc. Geod. Symp., vol. 102, edited by Y. Bock and N. Leppard, pp. 146-164, SpringerVerlag, New York, 1990a.

Lichten, S. M., Estimation and filtering for high precision GPS positioning applications, Man. Geod., 15, 159-176, $1990 \mathrm{~b}$.

Lichten, S. M., and J. S. Border, Strategies for high precision Global Positioning System orbit determination, J. Geophys. Res., 92, 12,751-12,762, 1987.

Lichten, S. M., and W. I. Bertiger, Demonstration of sub-meter GPS orbit determination and 1.5 parts in 108 three dimensional baseline accuracy, Bull. Geod., 63, 167-189, 1989.

Lindlohr, W., and D. Wells, GPS design using undifferenced carrier beat phase observations, Man. Geod., 10, 255-295, 1985.

Lorenzetti, E., and T. E. Tullis, Geodetic predictions of a strike slip fault model: Implications for intermediate and short term earthquake prediction, J. Geophys. Res., 94, 12,343-12,361, 1989.

Luyendyk, B. P., and J. S. Homafius, Neogene crustal rotation, fault slip and basin development in southern California, in Cenozoic Basin Development of Coastal California, Rubey vol. VI, edited by R. V. Ingersoll and W. G. Ernst, pp. 259-283, Prentice-Hall, Englewood Cliffs, N. J., 1987.

MacDoran, P., Satellite emission radio interferometric Earth surveying: SERIES GPS geodetic systems, Bull. Geod., 53, 117-138, 1979.

Malys, S., and P. A. Jensen, Geodetic point positioning with carrier beat phase data from the CASA Uno experiment, Geophys. Res. Lett., 17, 651-654, 1990.

McCaskill, T. B., and J. A. Buisson, On-orbit frequency stability of NAVSTAR GPS clocks and the importance of frequency stability to precise positioning, paper presented at First Intemational Symposium on Precise Positioning With Global Positioning System, Int. Assoc. of Geod., Rockville, Md., 1985.

Melboume, W. G., The case for ranging in GPS-based geodetic systems, First Intemational Symposium on Precise Positioning With Global Positioning System, Int. Assoc. of Geod., Rockville, Md., 1985.

Melboume, W. G., GPS-based geodesy tracking: Technology prospects for the year 2000, in Geodesy in the Year 2000, edited by J. Rundle, pp. 124-176, National Academy Press, Washington, D. C., 1990.

Melboume, W. G., R. Anderle, M. Feissel, R. King, D. McCarthy, D. Smith, B. Tapley, and R. Vicente, Project merit standards, Circ. 167, U.S. Nav. Observ., Washington, D. C., 1983.

Melbourne, W. G., R. Anderle, M. Feissel, R. King, D. McCarthy, D. Smith, B. Tapley, R. Vicente, Project merit standards, Circ. 167, Update 1, U.S. Nav. Observ., Washington, D. C., 1985.
Melosh, J., Nonlinear stress propagation in the Earth's upper mantle, J. Geophys. Res., 81, 5621-5632, 1976.

Melosh, J., Shear stress on the base of a lithospheric plate, Pure Appl. Geophys., 115, 429-439, 1977.

Minster, J. B., T. H. Jordan, B. H. Hager, D. C. Agnew, and L. H. Royden, Implications of precise positioning, in Geodesy in the Year 2000, edited by J. B. Rundle, pp. 23-45, National Academy Press, Washington, D. C., 1990.

Neilan, R., T. Dixon, T. Meehan, W. Melboume, J. Scheid, J. Kellogg, and J. Stowell, Operational aspects of CASA UNO '88-The first large scale international GPS geodetic network, IEEE Trans. Inst. Measur., 38, 648-651, 1989.

Neilan, R. E., W. G. Melbourne, and G. L. Mader, The development of a global GPS tracking system in support of space and ground-based GPS programs, in Global Positioning System: An Overview, Int. Assoc. Geod. Symp., vol. 102, edited by Y. Bock and N. Leppard, pp. 165-178, Springer-Verlag, New York, 1990.

Nur, A., H. Ron, and O. Scotti, Kinematics and mechanics of tectonic block rotations, in Slow Deformation and Transmission of Stress in the Earth, Geophys. Monogr. Ser., vol. 49, edited by S. C. Cohen and P. Vanicek, pp. 31-46, AGU, Washington, D. C., 1989.

Preston, R. A., R. Ergas, H. Hinteregger, C. Knight, D. Robertson, I. Shapiro, A. Whitney, A. Rogers, and T. Clark, Interferometric observations of an artificial satellite, Science, 178, 407-409, 1972.

Rabbel, W., and H. Schuh, The influence of atmospheric loading on VLBI experiments, J. Geophys., 59, 164-170, 1986.

Reeber, E. E., and J. R. Swope, On the correlation between total precipitable water in a column and absolute humidity at the surface, J. Appl. Meteorol., 11, 1322-1325, 1972.

Remondi, B. W., Global Positioning System carrier phase: Description and use, Bull. Geod., 59, 361-377, 1985.

Rizos, C., and A. Stolz, Force modelling for GPS satellite orbits, paper presented at First International Symposium on Precise Positioning With Global Positioning System, Int. Assoc. of Geod., Rockville, Md., 1985.

Robinson, S. E., The profile algorithm for microwave delay estimation from water vapor radiometer data, Radio Sci., 23, 401-408, 1988.

Rocken, C., J. Johnson, R. E. Neilan, M. Cerezo, J. Jordan, M. Falls, L. Nelson, R. Ware, and M. Hayes, The measurement of atmospheric water vapor: Radiometer comparison and spatial variation, IEEE Trans. Geosci. Remote Sens., in press, 1991.

Rocken, C., and C. M. Meertens, GPS antenna and receiver tests: Multipath reduction and mixed receiver baselines, Proceedings of Fifth International Symposium of Satellite Positioning, Las Cruces, New Mexico, pp. 375-385, 1989.

Rocken, C., and C. M. Meertens, Monitoring S/A and its effect on GPS geodesy (abstract), Eos Trans. AGU, 71, 1268, 1990.

Rosenblatt, D., Y. Bock, J.-B. Minster, and D. C. Agnew, The impact of recent Selective Availability on precise relative positioning with GPS (abstract), Eos Trans. AGU, 71, 1268, 1990.

Rundle, J. B., An approach to modelling present-day deformation in southern Califomia, J. Geophys. Res., 91, 1947-1959, 1986.

Savage, J. C., A dislocation model of strain accumulation and release at a subduction zone, $J$. Geophys. Res., 88, 4984-4996, 1983.

Savage, J. C., Equivalent strike-slip earthquake cycles in half space and lithosphere-asthenosphere Earth models, $J$. Geophys. Res., 95, 4873-4879, 1990.

Savage, J. C., and R. O. Burford, Geodetic determination of relative plate motion in central Califomia, J. Geophys. Res., 78, 832-845, 1973. 
Savage, J. C., and W. H. Prescott, Precision of Geodolite distance measurements, J. Geophys. Res., 78, 6001-6008, 1973.

Savage, J. C., and W. H. Prescott, Asthenosphere readjustment and the earthquake cycle, J. Geophys. Res., 83, 3369-3376, 1978.

Schaffrin, B., and E. W. Grafarend, Generating classes of equivalent linear models of nuisance parameter elimination: Application to GPS observations, Man. Geod., 11, 262-271, 1986.

Schutz, B. E., C. S. Ho, P. A. M. Abusali, and B. D. Tapley, CASA Uno orbit and baseline experiments, Geophys. Res. Lett., 17, 643-646, 1990.

Seidelman, P. K., 1980 IAU theory of nutation: The final report of the IAU working group on nutation, Celestial Mech., 27. 79-106, 1982.

Sibson, R. H., Continental fault structure and the shallow earthquake source, J. Geol. Soc. London, 140, 741-767, 1983.

Slade, M., and C. Yoder, 1960 Chile: New estimate of polar motion excitation, Geophys. Res. Lett., 16, 1193-1196, 1989.

Smith, D. E., D. C. Christodoulidis, R. Kolenkiewicz, P. Dunn, S. Klosko, M. Torence, S. Fricke, and S. Blackwell, A global geodetic reference frame from LAGEOS ranging, J. Geophys. Res., 90, 9221-9233, 1985.

Sovers, O. J., and J. Border, Observation model and parameter partials for the JPL GPS geodetic modeling software "GPSOMC," JPL Publ., 87-21, Rev. 2, 1990.

Spence, D. A., and D. L. Turcotte, Viscoelastic relaxation of cyclic displacement on the San Andreas fault, Proc. R. Soc. London, Ser. A, 365, 121-144, 1979.

Spilker, J. J., GPS signal structure and performance characteristics, Nav. J. Inst. Nav., 25, 121-146, 1978.

Tapley, B. D., B. E. Schutz, and R. J. Eanes, Station coordinates, baselines and earth rotation from LAGEOS laser ranging: 1976-1984, J. Geophys. Res., 90, 9235-9248, 1985.

Thatcher, W., Non-linear strain buildup and the earthquake cycle on the San Andreas fault, J. Geophys. Res., 88, 5893-5902, 1983.

Thatcher, W., and J. B. Rundle, A viscoelastic coupling model for the cyclic deformation due to periodically repeated earthquakes at subduction zones, J. Geophys. Res., 89, 7631-7640, 1984.

Thompson, A. R., J. M. Moran, and G. W. Swenson, Interferometry and Synthesis in Radio Astronomy, 534 pp., Wiley-Interscience, New York, 1986.

Thornton, C. L., and G. J. Bierman, UDU ${ }^{T}$ covariance factorization for Kalman filtering, in Control and Dynamic Systems, Advances in Theory and Applications, vol. 16, edited by C. T. Leondes, pp. 177-248, Academic, Troy, Mo., 1980.
Tralli, D. M., and T. H. Dixon, A few parts in $10^{8}$ geodetic baseline repeatability in the Gulf of Califomia using the Global Positioning System, Geophys. Res. Lett., 15, 353-356, 1988.

Tralli, D. M., and S. M. Lichten, Stochastic estimation of tropospheric path delays in Global Positioning System geodetic measurements, Bull. Geod., 64, 127-159, 1990.

Tralli, D. M., T. H. Dixon, and S. A. Stephens, The effect of wet tropospheric path delays on estimation of geodetic baselines in the Gulf of California using the Global Positioning System, $J$. Geophys. Res., 93, 6545-6557, 1988.

Turcotte, D. L., J. Y. Liu, and F. H. Kulhawy, The role of an intracrustal asthenosphere on the behavior of major strike-slip faults, J. Geophys. Res., 89, 5801-5816, 1984.

Wahr, J. M., The forced nutations of an elliptical, rotating, elastic and oceanless Earth, Geophys. J. R. Astron. Soc., 64, 705-727, 1981.

Wahr, J. M., The Earth's rotation rate, Ann. Rev. Earth Planet. Sci., 16, 231-249, 1988.

Ware, R. H., C. Rocken, and J. B. Snider, Experimental verification of improved GPS-measured baseline repeatability using water vapor radiometer corrections, IEEE Trans. Geosci. Remote Sens., GE-23, 467-473, 1985.

Webb, F., Geodetic measurement of deformation in the offshore of southem California, Ph.D. thesis, Calif. Inst. of Technol., Pasadena, 1990.

Weldon, R., and E. Humphreys, A kinematic model of southem California, Tectonics, 5, 33-48, 1986.

Wu, S. C., W. I. Bertiger, and J. T. Wu, Minimizing selective availability error on Topex GPS measurements, paper presented at American Institute of Aeronautics and Astronautics Conference, Portland, Oreg., 1990.

Wubbena, G., Software developments for geodetic positioning with GPS using TI-4100 code and carrier measurements, paper presented at First International Symposium on Precise Positioning With Global Positioning System, Int. Assoc. of Geod., Rockville, Md., 1985.

Wyatt, F., Displacements of surface monuments: Vertical motion, J. Geophys. Res., 94, 1655-1664, 1989.

Young, L. E., R. Neilan, N and F. Bletzacker, GPS satellite multipath: An experimental investigation, paper presented at First International Symposium on Precise Positioning With Global Positioning System, Int. Assoc. of Geod., Rockville, Md., 1985.

T. H. Dixon, Jet Propulsion Laboratory, Division of Earth and Planetary Sciences, 4800 Oak Grove Drive, Pasadena, CA 91109. 